\title{
Validation of global gyrokinetic simulations in stellarator configurations
}

\author{
E. Sánchez ${ }^{1}$, T. Estrada ${ }^{1}$, J. L. Velasco ${ }^{1}$, I. Calvo ${ }^{1}$, A. \\ Cappa $^{1}$, A. Alonso ${ }^{1}$, J. M. García-Regaña ${ }^{1}$, R. Kleiber ${ }^{2}$, J. \\ Riemann $^{2}$ and the TJ-II team \\ ${ }^{1}$ Laboratorio Nacional de Fusión / CIEMAT. Avda Complutense 40, 28040, \\ Madrid, Spain \\ ${ }^{2}$ Max-Planck Insitut für Plasmaphysik, Greifswald, Germany. \\ E-mail: edi.sanchez@ciemat.es
}

10 June 2019

\begin{abstract}
In this paper, recent simulations carried out with the global gyrokinetic code EUTERPE in configurations of the TJ-II stellarator are presented. The simulations are compared with experimental measurements from the Doppler Reflectometry (DR) system in dedicated experiments carried out in TJ-II. Electron-driven modes are found unstable in the plasma edge region in linear simulations. The range of unstable wavenumbers and the radial location of maximum instability found in simulations are consistent with the experimental density fluctuations spectra and their variation with radius. A qualitative agreement is found between the poloidal propagation of unstable modes found in simulations and the perpendicular velocities measured by the DR system. No dependency of the power spectra with the bulk ion mass is observed in simulations or experimentally, which is consistent with the electron-driven modes found unstable in simulations. Instabilities are localized toroidal and poloidally in simulations, the location of maximum instability being affected by the rotational transform. A systematic difference is found between the density fluctuation spectra measured by the DR system at poloidally separated positions on the same flux-surface, which is also affected by the rotational transform. However, a discrepancy between the location of maximum fluctuations in simulations and experiments is found so far.
\end{abstract}

Keywords: plasma turbulence, gyrokinetic simulations, EUTERPE 


\section{INTRODUCTION}

Gyrokinetics [1-5] constitutes the proper theoretical framework to study turbulence in magnetized plasmas from first principles. The theoretical study and the numerical simulation of micro-instabilities and turbulence in a tokamak are largely facilitated by its axisymmetry which makes all the field lines in a flux surface equivalent. The simulations can be carried out in a reduced domain, flux-tube, following the magnetic field line, covering just a poloidal turn, and usually with periodic boundary conditions, thus largely reducing the computational resources required. In stellarators, the lack of symmetry implies a three-dimensional dependence of many equilibrium quantities affecting micro-instabilities, such as the magnetic field line curvature, the local magnetic shear or the location of magnetic wells. As a consequence, different flux-tubes in a flux surface are not equivalent to each other in general [6]. In stellarator simulations, the instabilities appear localized both poloidally and toroidally on the flux surface [7, 8, 9, 10, which is a distinct feature of stellarators largely affecting the turbulent transport level [11, 12. This feature would be susceptible of experimental verification, although this is a challenging task, as it requires good spatial resolution and probing several regions of the flux surface. The influence of radial electric fields, which play a key role in stellarator confinement, is affected by the magnetic geometry and more involved in stellarators than in the tokamak counterpart 13 , 14. Furthermore, the linear response of zonal flows (ZF), which are considered an important ingredient in the saturation of plasma turbulence, shows distinct features in stellarators as compared to tokamaks [15. The turbulence-ZF interaction is largely affected by the configuration in several ways and different saturation mechanisms, with different roles of ZFs, can appear depending on the magnetic configuration [16]. Because of all these reasons, the minimum appropriate computational domain to be used in simulations for stellarators should cover, in general, at least the full flux surface. In some particular cases, the use of extended flux tube domains have allowed to obtain similar results between different flux tubes [18. However, this can not be considered a general rule.

Simulation codes need to be verified against other codes and theoretical calculations, and finally validated against experiments to ensure that the models implemented in them properly describe the physics. There is a variety of simulation codes based on the gyrokinetic formalism for tokamaks and a significant effort is being developed for their validation. Fewer codes are available for stellarators, however, and many of them are based on the direct port of the flux-tube approximation to the stellarator geometry, which is far from optimum. The validation task is consequently much less mature than in the tokamak counterpart. Few codes are suited to be used in the full flux surface, or global, computational domains in stellarators nowadays.

EUTERPE [17 is one such code, which was developed from the beginning with the aim of performing global simulations of instabilities in 3D geometry. A long-term effort for verifying and validating this code is ongoing. The linear relaxation of ZFs in stellarators has been extensively studied with EUTERPE in many stellarator configurations [19, 20]. The code has been verified by comparing the properties of linear ZF relaxation obtained in simulations with both other gyrokinetic codes and analytical estimations [21, 22]. Furthermore, these calculations were validated against experimental measurements obtained during pellet injection experiments carried out in TJII, in which an oscillatory relaxation of potential perturbations was observed for the first time 23, 24.

Withing this validation effort, the comparison of simulations with experimental turbulence measurements in both TJ-II and W7-X stellarators is under way. Two Doppler Reflectometry (DR) systems, looking at different cross sections, have been installed in W7-X for this purpose. In TJ-II the DR system is equipped with a steerable mirror that allows probing different positions over the same flux surface. Dedicated experiments have been carried out in TJ-II for the validation of simulations with experimental turbulence measurements from DR. The results of this comparison are presented in this work. The comparison between simulation and experiments has been pursued at different levels of detail, including the density fluctuation level and wavenumber spectra and their radial variation, and particular attention was paid to the localization of instabilities along the flux surface taking advantage of the capabilities of this DR system.

The rest of the paper is organized as follows. The code EUTERPE is presented in section 2, the setup used in the experiments is described in section 3 and the results from simulations are presented in section 4. In section 5, the results are summarized and some conclusions are drawn.

\section{THE CODE EUTERPE}

EUTERPE is a global gyrokinetic code developed with the aim of simulating plasmas in three dimensional geometries [17, 25]. A particle-in-cell scheme is used in the code, the distribution function being discretized using markers.

The gyro-averaged kinetic equation

$$
\frac{\partial f_{a}}{\partial t}+\dot{\mathbf{R}_{\mathbf{a}}} \frac{\partial f_{a}}{\partial \mathbf{R}}+v_{a \|} \frac{\partial f_{a}}{\partial v_{\|}}=\sum_{b} C\left(f_{a}, f_{b}\right)
$$


is solved for the distribution function $f_{a}$ of up to three kinetic species.

The characteristic curves $\dot{\mathbf{R}}_{\mathbf{a}}=\frac{d \mathbf{R}_{\mathbf{a}}}{d t}$ and $v_{a \|}^{\cdot}=$ $\frac{d v_{a \|}}{d t}$ are the equations of motion for the markers, which can be separated into an unperturbed (labeled as ${ }^{0}$ ) and a perturbed part (labeled as ${ }^{1}$ ) as $\dot{\mathbf{R}_{\mathbf{a}}}=\dot{\mathbf{R}}_{\mathbf{a}}^{\mathbf{0}}+\dot{\mathbf{R}}_{\mathbf{a}}^{1}$ and $\dot{v}_{\|}=v_{a \|}^{\dot{0}}+v_{a \|}^{\dot{i}}$. In the electrostatic approximation here used, these equations can be written as:

$$
\begin{aligned}
& \dot{\mathbf{R}_{a}^{0}}=v_{a \|} \mathbf{b}+\frac{\mu_{a} B+v_{a \|}^{2}}{B_{a \|}^{*} \Omega_{a}} \mathbf{b} \times \nabla B \\
& +\frac{v_{a \|}^{2}}{B_{a \|}^{*} \Omega_{a}}(\nabla \times \mathbf{B})_{\perp}-\frac{\nabla \varphi_{L W} \times \mathbf{b}}{B_{a \|}^{*}}, \\
& v_{a \|}^{\dot{0}}=-\mu_{a}\left[\mathbf{b}+\frac{v_{a \|}}{B_{a \|}^{*} \Omega_{a}}(\nabla \times \mathbf{B})_{\perp}\right] \cdot \nabla \mathbf{B} \\
& -\frac{q_{a}}{m_{a}}\left[\mathbf{b}+\frac{v_{a \|}\left(\mathbf{b} \times \nabla B+(\nabla \times \mathbf{B})_{\perp}\right)}{B_{a \|}^{*} \Omega_{a}}\right] \cdot \nabla \varphi_{L W}, \\
& \dot{\mathbf{R}}_{a}^{1}=-\frac{\nabla\{\varphi\} \times \mathbf{b}}{B_{a \|}^{*}}, \\
& v_{a \|}^{\mathrm{i}}=\frac{-q_{a}}{m_{a}}\left[\mathbf{b}+\frac{v_{a \|}\left(\mathbf{b} \times \nabla B+(\nabla \times \mathbf{B})_{\perp}\right)}{B_{a \|}^{*} \Omega_{a}}\right] \cdot \nabla\{\varphi\} .
\end{aligned}
$$

The magnetic moment per unit mass, $\mu_{a}$, is a constant of motion $\left(\dot{\mu}_{a}=0\right) ; q_{a}$ and $m_{a}$ are the charge and mass of the species a, respectively, $\Omega_{a}=q_{a} B / m_{a}$ is the cyclotron frequency, $\mathbf{b}=\mathbf{B} / B$ is the unit vector in the magnetic field $(\mathbf{B})$ direction and $B_{a \|}^{*}=\mathbf{b} \cdot \mathbf{B}_{\mathbf{a}}^{*}$, with $\mathbf{B}_{\mathbf{a}}^{*}=\mathbf{B}+\frac{m_{a} v_{a \|}}{q_{a}} \nabla \times \mathbf{b} .\{\varphi\}$ is the gyro-averaged potential introduced in Ref. [3. $\nabla \varphi_{L W}$ represents the ambient, long-wavelength radial electric field, which in stellarators is, in general, determined by neoclassical processes [27.

The $\delta f$ (or control variate) method is used, so that the distribution function is separated into an equilibrium function (local Maxwellian), $F_{M a}$, plus a time-dependent perturbation as $f_{a}\left(R, v_{\|}, \mu, t\right)=$ $F_{M a}(R, v \|, \mu)+\delta f\left(R, v_{\|}, \mu, t\right)$. This separation allows to write an equation for the $\delta f$, which is non-linear,

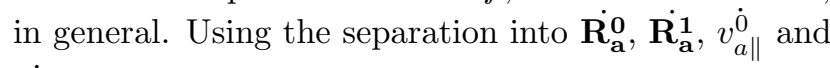
$v_{a \|}^{\mathrm{i}}$ depicted in Equations (2) to $(5,5)$ a linearized kinetic equation for $\delta f_{a}$ can be written, as:

$$
\begin{aligned}
\frac{\partial \delta f_{a}}{\partial t}+ & \dot{\mathbf{R}_{\mathbf{a}}^{0}} \frac{\partial \delta f_{a}}{\partial \mathbf{R}}+v_{\| a}^{\dot{0}} \frac{\partial \delta f_{a}}{\partial v_{\|}}= \\
& \quad \dot{\mathbf{R}_{\mathbf{a}}^{\mathbf{1}}} \frac{\partial F_{M a}}{\partial \mathbf{R}}-v_{\| a}^{\mathrm{i}} \frac{\partial F_{M a}}{\partial v_{\|}}+\sum_{b} C\left(\delta f_{a}, f_{b}\right) .
\end{aligned}
$$

In this work, we consider two kinetic species, ions and electrons, and the collisions are taken into account using a pitch-angle scattering collision operator [26].

The kinetic equation (1), or its linearized version (6) here used, is solved together with the quasi neutrality equation (QNE), which for kinetic ions and electrons can be written as:

$$
q_{i}\left\{n_{i}\right\}-n_{e}=\frac{q_{i}^{2} n_{i 0}}{T_{i 0}}\left[\Gamma_{0}\left(k_{\perp}^{2} \rho_{i}^{2}\right)-1\right] \varphi,
$$

where the "0" subindex refers to the equilibrium density and temperature.

A Padé approximation is used for the Gamma function, $\Gamma_{0}=e^{-x} I_{0}(x) \approx 1 /(1+x)$, with $x=k_{\perp}^{2} \rho_{i}^{2}$ and $I_{0}$ being the modified Bessel function, $k_{\perp}$ the perpendicular wavenumber and $\rho_{i}=\sqrt{T_{i} m_{i}} / e B$ the thermal ion Larmor radius. Then, the QNE gives a field equation in the form:

$$
q_{i}\left\{n_{i}\right\}-n_{e}=-\nabla \frac{m_{i} n_{0}}{B^{2}} \nabla_{\perp} \varphi+\nabla \rho_{i}^{2} \nabla_{\perp}\left(q_{i}\left\{n_{i}\right\}-n_{e}\right) .
$$

It can be shown that the Padé approximation is good up to scales $k_{\perp}^{2} \rho_{i}^{2} \sim 2$ 团 while at the same time preserves a quadratic form for the operator acting on the potential in the QNE, which is convenient from the numerical point of view.

The gyro-average ( \{\} operation) appearing in Eqs. (4), (5), (7) and (8) is in practice computed with values at a finite number of points along the gyroring. For small wavenumbers this average is computed with just 4 points (set as the default value in the code), which can be shown to be valid for $k_{\perp} \rho_{i}<2$ [28, 29].

The PEST1 magnetic coordinates 30 $(s, \theta, \phi)$ are used for resolving the potential, $\phi$, with $s$ being the normalized toroidal flux, used as radial coordinate and $\theta$ and $\phi$ the poloidal and toroidal magnetic coordinates angles, respectively. Cylindrical coordinates are used for evolving the markers.

The magnetic field, not evolved in these electrostatic simulations, is obtained from a MHD calculation with the code VMEC 31]

More details about the code can be found in Refs [8, 25, 32, 33, 34.

\section{EXPERIMENTAL SET-UP}

TJ-II is a heliac stellarator operated in the National Fusion Laboratory, in CIEMAT, whose main parameters are: major radius $R=1.5 \mathrm{~m}$, minor radius $a=0.22 \mathrm{~m}$ and maximum toroidal magnetic field $B_{T}=1.2 \mathrm{~T}$. A wide range of rotational transforms, roughly from $t=0.95$ to $t=2.5$, with very small magnetic shear, can be explored. The plasmas are heated by ECRH using two gyrotrons at $53.2 \mathrm{GHz}$ (2nd harmonic) with X-mode polarization and a nominal power of $300 \mathrm{~kW}$ each. The radial location of ECRH power deposition can be changed by means of a mirror located at the end of the transmission line 35. In addition,

$\ddagger$ For scales $k_{\perp}^{2} \rho_{i}^{2}>2$ the Padé approximation has the effect of over-damping the modes at these scales. 

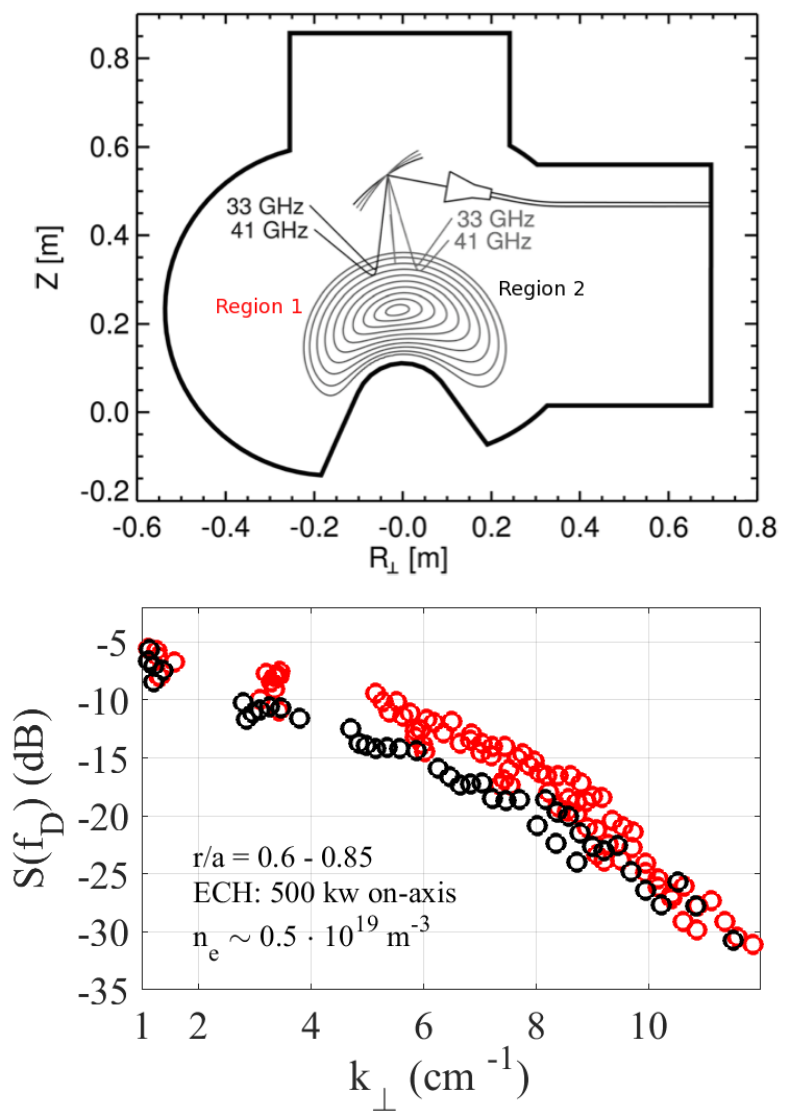

Figure 1. Schematic view of the DR system installed in TJ-II (top) showing the two poloidal regions of measurement (Region_1 and Region_2) and the $\tilde{n}$ wavenumber spectra (bottom) measured at both regions in the plasma scenario with peaked electron temperature profile (see text for details).

two neutral beam injectors (NBI) with $700 \mathrm{~kW}$ nominal port-through power per injector are available [36].

An optimized Doppler Reflectometry (DR) system is in operation in TJ-II, which allows to measure density fluctuations $(\tilde{n})$ in the plasma edge in a range of perpendicular wavenumbers $k_{\perp}=1-14 \mathrm{~cm}^{-1}$ [37. In addition to the fluctuations, the radial electric field $\left(E_{r}\right)$ can also be measured (assuming that the plasma fluctuations velocity is dominated by the $\mathbf{E} \times \mathbf{B}$ drift). The radial location of measurement is determined by the position at which the microwave beam is reflected, which depends on the plasma density and the magnetic field. The reflection location can be changed by modifying (even during the discharge) the frequency of the incident wave, thus providing measurements in the region of normalized radii $r / a=0.55$ to $r / a=0.95$, depending on the plasma conditions. The system is also equipped with a steerable mirror which allows probing different wavenumbers when it is slightly rotated. The wavenumber and measurement location is obtained using the ray tracing code TRUBA [38] with the density profile as input for it. The change in the mirror angle has to be done between discharges; then a set of similar discharges are required in order to explore the wavenumber space and construct a spectrum of density fluctuations. Larger movements of the mirror allow probing different poloidal regions (see figure 1 . The DR system had previously been routinely operated to measure at just one poloidal region of the plasma (Region_1). With the goal of characterizing the turbulence localization, in the experiments described in this work, the DR system was operated to measure at two poloidally separated locations (Region_1 and Region_2), thus unraveling interesting results [39]. An example of density fluctuation spectra measured at two poloidal regions is shown in figure 1 .

By changing the ECRH heating power and deposition location, plasmas with different density and temperature profiles were obtained. Basically, three plasma scenarios have been produced, which we name after the shape of the electron temperature profile. In the first scenario, which we name peaked, full ECRH power was deposited on-axis, which produced plasmas with line-averaged densities in the range $5 \cdot 10^{18} \mathrm{~m}^{-3}$ and peaked $T_{e}$ profiles. A second scenario (broad), with similar line-averaged densities but less peaked $T_{e}$, was generated with full ECRH power deposited offaxis (at $r / a \sim 0.64$ ) and finally, using half ECRH power deposited off-axis (at $r / a \sim 0.4$ ), higher density plasmas $\left(\bar{n} \sim 10^{19} \mathrm{~m}^{-3}\right)$ were obtained. In this third scenario (hollow), the off-axis deposition of the heating power combined with high densities, which hinder the propagation of ECRH and the heating of the core, produce a hollow electron temperature profile.

In both the scenarios peaked and broad the plasma is in the electron root regime with the electric field being positive, while in the hollow scenario, with higher density, the plasma has transited to the ion root regime 41] and the radial electric field is negative.

The density and temperature profiles for these scenarios were reconstructed in an integrated manner 42], which uses the Thomson scattering [44, Neutral Particle Analyzer (NPA) [45], interferometer, AM reflectometry 43, Helium beam 46, 47, 48, and electron cyclotron emission diagnostics 49, when available and takes into account the respective calibration factors and errors of all the diagnostics involved. The density and temperature profiles for three discharges (\#42759, \#42779 and \#45429) representative of each scenario are shown in figure 2 . The ion temperature measurements are obtained from the NPA, which provides only two central measurements per discharge with time resolution. The ion temperature profile is constructed using the central measurements available and some general recipes [50] empirically derived, and then the uncertainties in this profile are large. Note, however, that, as the 


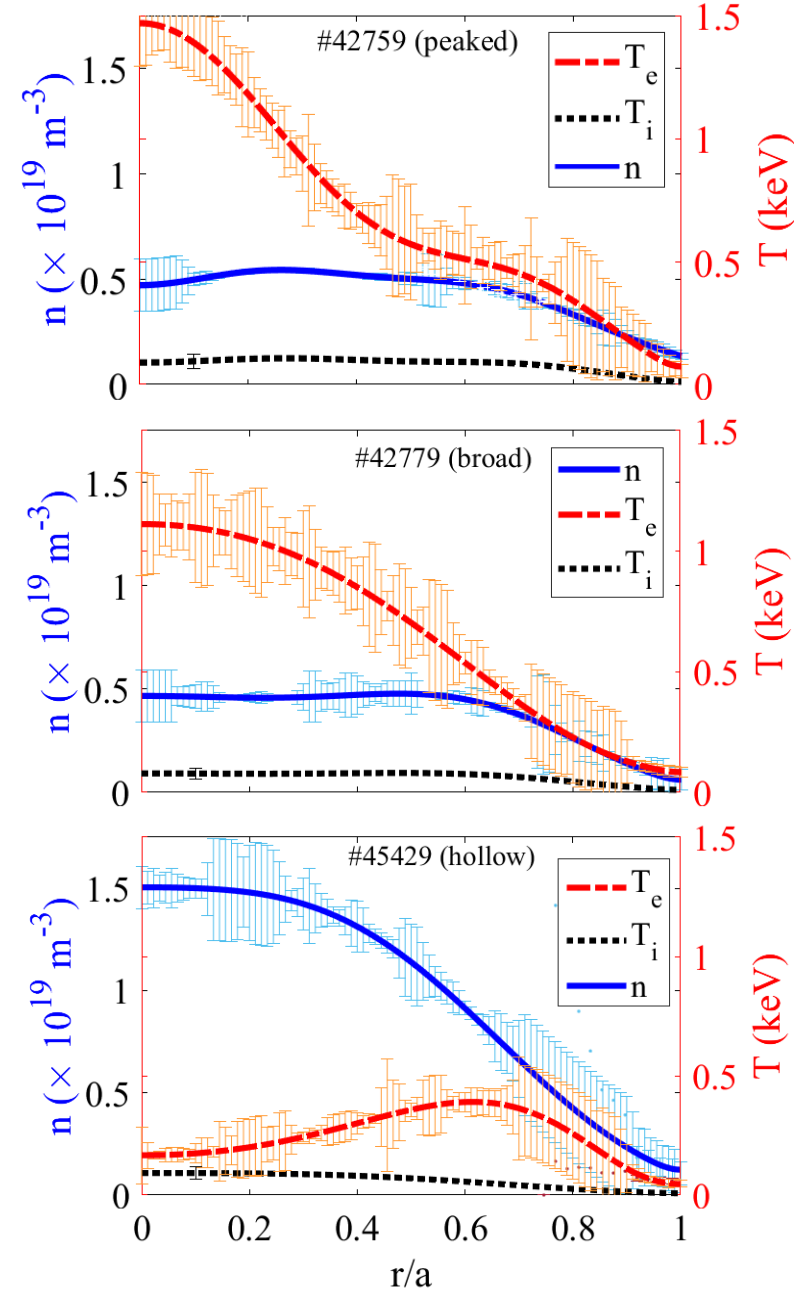

Figure 2. Density and temperature profiles for three discharges representative of the three plasma scenarios described in this work: \#42759 (peaked), \#42779 (broad) and \#45429 (hollow). The uncertainty in the measurements derived from the various diagnostics is captured by the bayesian profile reconstruction and shown in the figure with the error bars.

instabilities that are identified in this work are driven mainly by electrons, the $T_{i}$ profile is not as relevant as the density and electron temperature profiles (see Sections 4.2 to 4.4. For these plasma scenarios, in the radial region of interest, $0.65<r / a<0.9$, the electrons are in the banana-plateau regime with $\nu_{e *}=\nu_{e} q R / v_{\text {the }} / \epsilon^{3 / 2}=0.3-0.6$ and $\nu_{e *}=0.3-1$ for the scenarios peaked and broad, respectively and $\nu_{e *}=0.8-1.6$ in the more collisional hollow scenario. $\nu_{e}$ is the electron collision frequency, $\epsilon=r / R$ is the aspect ratio and $v_{t h e}$ is the electron thermal velocity.

The radial electric field was measured by DR for all the scenarios, which compared well with independent measurement with the Heavy Ion Beam Probe diagnostic [51, and showed rough agreement with neoclassical calculations using DKES [52]. For each plasma scenario, a series of discharges was carried out under similar conditions in the standard magnetic configuration, which allowed to obtain the density fluctuations spectra.

Both Hydrogen and Deuterium plasmas have been conducted to study the effect of ion mass on the instabilities and, in addition, several magnetic configurations were used to explore the influence of the rotational transform. The experimental results obtained with the DR system are presented in detail in a separate work [39] to which we refer the reader for details.

\section{SIMULATION RESULTS AND COMPARISON WITH EXPERIMENTS}

Global gyrokinetic simulations were carried out with EUTERPE for the three plasma scenarios previously described using the density, temperature and $E_{r}$ profiles for each representative discharge of the corresponding series as input. The simulations are restricted to the radial region $r / a>0.32$.

A linear stability analysis for these plasmas was carried out both with and without taking into account collisions and also with and without accounting for the radial electric field. The simulations without including $E_{r}$ or collisions allow us to analyze the influence of density and temperature gradients alone in driving instabilities, while simulations including $E_{r}$ and collisions are more realistic and more comparable to the experimental situation. The simulations are carried out using fully kinetic electrons and ions, with a spatial resolution of $n_{s} \times n_{\theta} \times n_{\phi}=64 \times 256 \times 128$. With this resolution the simulations are covering roughly a wavenumber range $k_{\theta} \rho_{i}<1$ in the outer region $(r / a>0.6)$ of the plasma, with $k_{\theta}<10 \mathrm{~cm}^{-1}$ at $r / a \sim 0.9$ and $k_{\theta}<12 \mathrm{~cm}^{-1}$ at $r / a \sim 0.7$. As the wavenumber resolution is kept constant with radius, at the inner positions the simulations cover wavenumbers beyond this limits and even larger than $k_{\perp} \rho_{i}=1$. Note that $k_{\theta} \lesssim k_{\perp}$. This wavenumber range covers the range of measurements of density fluctuations spectra provided by the DR system, $\left(1<k_{\perp}<12 \mathrm{~cm}^{-1}\right)$ for $r / a>0.6$ (see figure 1 and Ref. [39]). With respect to the radial resolution, as in these simulations the radial domain is proportionally divided in the normalized toroidal flux, the resolution changes with radius. In the radial region of interest $r / a=0.65-0.9$, the radial resolution is $\Delta r \simeq 2.4-2 \mathrm{~mm}$, or in terms of the ion Larmor radius $\Delta r / \rho_{i}<3$. Simulations with larger radial resolution $n_{s}=100,128$ were carried out and the results were very similar to those with $n_{s}=64$ : with very similar spectra of unstable modes and growth rates differing less than $1 \%$, which indicates that numerical convergence in the radial resolution was reached at $n_{s}=64$. The convergence with the 
number of markers, $N_{M}$, was also studied in a set of simulations with $N_{M}=100,150$ and 300 million markers, showing that for $N_{M}=150 \cdot 10^{6}$ the results are well converged. The simulations shown in this work used $N_{M}=150 \cdot 10^{6}$.

In the simulations presented in this work, the inverse normalized ion Larmor radius is $1 / \rho^{*}=a / \rho_{i} \simeq$ $250-350$ and the ratio of ion Larmor radius to the radial scale lengths of density and temperature profiles are always $L_{T_{i}} / \rho_{i}>35, L_{T_{e}} / \rho_{i}>35, L_{T_{n}} / \rho_{i}>50$ (see Figure 4 for values of $L_{n}, L_{T_{i}}$ and $\mathrm{L}_{\mathrm{T}_{\mathrm{e}}}$ ). These values indicate that non-local effects can be relevant in these simulations [0].

Note that these linear simulations do not provide a proper density or potential wavenumber spectrum, but a spectrum of unstable modes, which would define the real spectrum only after non-linear saturation. However, the spectrum of unstable modes is related to the experimentally measurable one: the unstable modes define the region of injection of energy in the fluctuations wavenumber spectrum and they are expected to be close to the knee that is commonly found in experimental spectra (see figure 1-bottom and 39] for more details), which appears as a consequence of the nonlinear saturation, involving energy cascades both upwards and downwards from the point of injection [53]. In the cases under study, the knee of the experimental spectra in all three scenarios is found at $k_{\perp} \sim 6-8 \mathrm{~cm}^{-1}$ [39] which is in rough agreement with the most unstable modes found in simulations (see figure 3 and table 11. In the broad scenario, the most unstable mode in the simulation is $m=225$, corresponding roughly to $k_{\theta}=9.5 \mathrm{~cm}^{-1}$, however, there are several modes with comparable growth rates around $120<m<160$, corresponding to $k_{\theta} \sim 6 \mathrm{~cm}^{-1}$, too.

In figure 3, the amplitude of the potential spectrum at toroidal angle $\phi=0$ (normalized to its maximum value) is shown versus the normalized radius $(r / a)$ and the poloidal mode number $(m)$ for linear simulations including the experimental density, temperature and $E_{r}$ profiles and taking into account the collisions for the reference discharges whose profiles are shown in figure 2 Color lines over the plots show the location of modes with specific wavenumbers $\left(k_{\theta}=\right.$ $\left.2,4,6,8,10 \mathrm{~cm}^{-1}\right)$. In these plots, the wavenumber is calculated as $k_{\theta}=m\left|\nabla \theta_{\text {min }}\right|$, with $\left|\nabla \theta_{\text {min }}\right|$ being the minimum value of $|\nabla \theta|$ on the flux surface, which is suggested by the fact that the unstable modes appear located in regions where $|\nabla \theta|$ is close to its minimum over the flux surface $[9]$.

Unstable electrostatic modes are found whose degree of instability is maximum in the outer region of the plasma $(r / a>0.6)$, where both the density and electron temperature gradients are negative, with

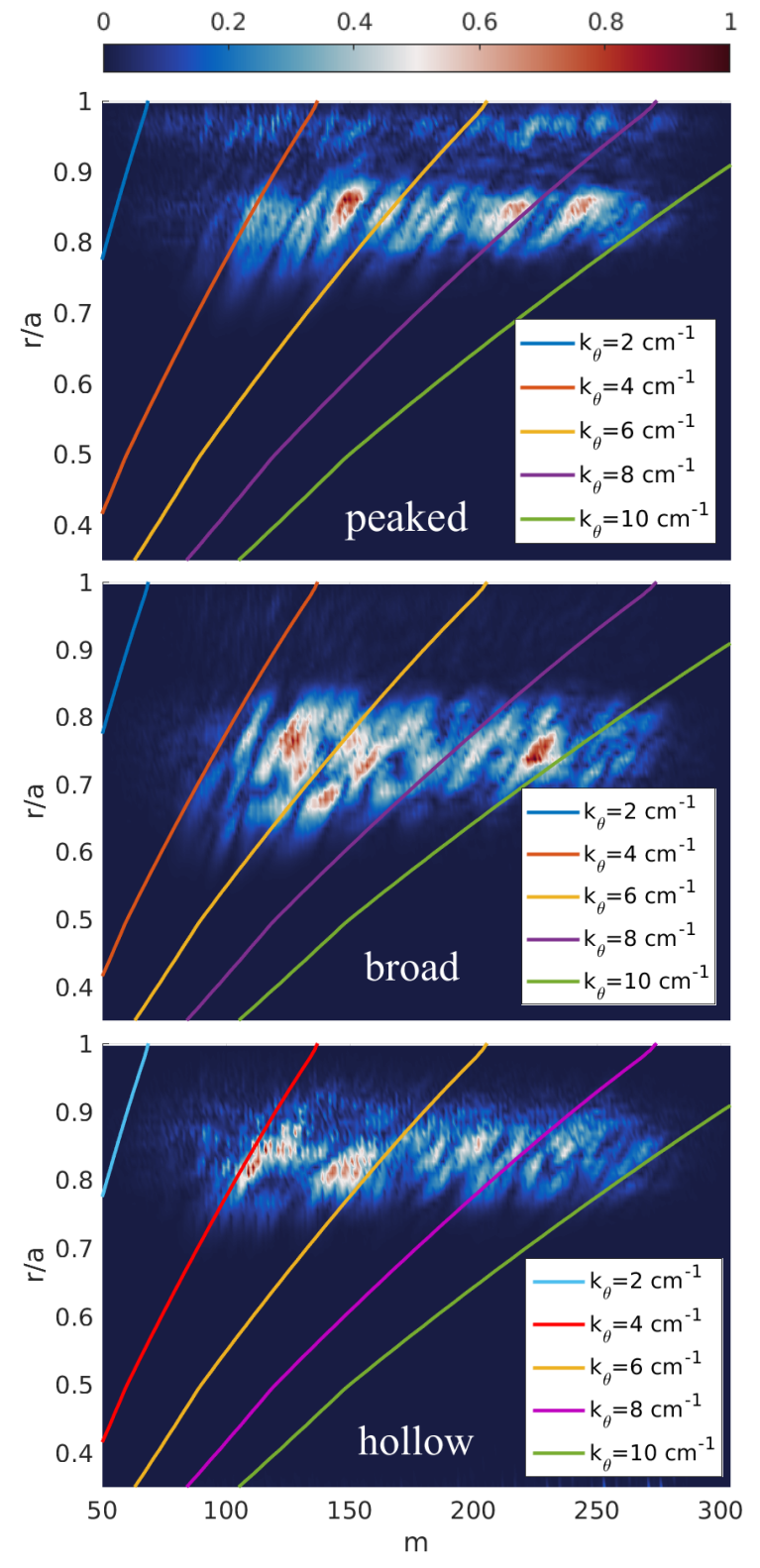

Figure 3. Spectral amplitude of the perturbed potential (normalized to its maximum value) in a set of linear collisional simulations using experimental density, temperature (figure 2) and electric field profiles for the three plasma scenarios. The horizontal axis is the poloidal-like mode number, $\mathrm{m}$, associated to the $\theta^{*}$ PEST1 magnetic coordinate.

maximum growth rates in the range $\gamma=6.5-8 \cdot 10^{5} \mathrm{~s}^{-1}$ and real frequencies in the range $\omega=15-40 \cdot 10^{5} \mathrm{~s}^{-1}$. Unstable modes, with smaller growth rates, are also found in the core region $(r / a \sim 0.35)$ in the peaked scenario but we will concentrate on the edge region, where the simulation results can be compared to the Doppler reflectometry measurements. These values of growth rates and frequencies correspond to the simulations including both $E_{r}$ and collisions shown in figure 3 For simulations without them the growth 
Table 1. Mode number, $m$, wavenumber, $k_{\theta}$, frequency, $\omega$, growth rate, $\gamma$ and radial location of maximum amplitude, $r / a$, of the most unstable mode found in the simulations studied in this work. $\omega$ and $\gamma$ are in $10^{5} \mathrm{~s}^{-1}$ units and $k_{\theta}=m \nabla \theta_{\min }$ is given in $\mathrm{cm}^{-1}$. In the second column the value of the electric field at the position of maximum instability is given, in $\mathrm{kV} / \mathrm{m}$, for the simulations including collisions and radial electric field.

\begin{tabular}{|c|c|c|c|c|c|c|c|}
\hline \multirow{2}{*}{ scenario } & \multirow{2}{*}{$\begin{array}{c}E_{r} \\
\text { col }\end{array}$} & \multicolumn{5}{|c|}{ most unstable mode } & \multirow{2}{*}{ Figs. } \\
\hline & & $m$ & $k_{\theta}$ & $\omega$ & $\gamma$ & $r / a$ & \\
\hline \multirow{2}{*}{ peaked,H } & 2.5 & 12 & 5.1 & 6.5 & 6.8 & .87 & \\
\hline & - & 247 & 8.6 & 2.1 & 8.5 & .87 & $7 \mid 8$ \\
\hline \multirow{2}{*}{ broac } & 3.5 & 225 & 9.5 & 37.7 & 6.9 & .74 & \multirow{2}{*}{\begin{tabular}{|l|l|l|}
3 & 10 & 13 \\
7 & & 13 \\
\end{tabular}} \\
\hline & - & 212 & 8.9 & 3.1 & 7.4 & .74 & \\
\hline \multirow{2}{*}{ hollo } & -3 & 116 & 4.1 & 25 & 7.7 & .85 & \multirow{2}{*}{\begin{tabular}{|l|l|l}
3 & 10 & 1 \\
7 & 8
\end{tabular}} \\
\hline & - & 246 & 8.8 & 3.5 & 9.1 & .86 & \\
\hline & 2.5 & 146 & 5.1 & 19 & 4.7 & 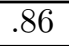 & 12 \\
\hline eaked, $\mathrm{H}, t$ & 2.5 & 161 & 5.6 & 25.4 & 6.8 & .87 & \\
\hline
\end{tabular}

rates are slightly larger, as both ingredients contribute to stabilizing the modes, and the frequencies are significantly smaller as the $E_{r}$ produces a Doppler shift which increases the frequencies (see table 11). The growth rates and frequencies of the unstable modes found in all the simulations in this work are given in Table 1. In this table, the mode number, $m$, the poloidal wavenumber, $k_{\theta}$, the frequency, $\omega$, the growth rate, $\gamma$, and the radial location of maximum amplitude for the most unstable mode are given. It is also indicated whether the $\mathrm{NC}$ background electric field and collisions are considered in the simulation, the value of the background electric field at the radial position of maximum instability, when applicable, and the figures corresponding to each case.

The character of the unstable modes found in these simulations will be analyzed in some detail in the next sections. They appear to be driven mainly by electrons, as will be shown in section 4.2. This was already expected because no ITG modes were found in adiabatic electron simulations with experimental typical profiles from TJ-II discharges carried out in previous works $[9,55]$. Next, we analyze in some detail the radial location of instabilities.

\subsection{Radial location of instabilities}

The change in the heating power and deposition location from scenario to scenario modifies significantly the density and temperature profiles (see figure 2), and consequently, their gradients are also modified. In figure 4 the normalized scale lengths of density and electron temperature profiles $\left(L_{n}=n(\partial n / \partial r)^{-1}\right.$ and $\left.L_{T_{e}}=T_{e}\left(\partial T_{e} / \partial r\right)^{-1}\right)$ are shown together with the parameter $\eta_{e}=L_{n} / L_{T_{e}}$ for the reference discharges of the three plasma scenarios.

The change in profile gradients displaces radially
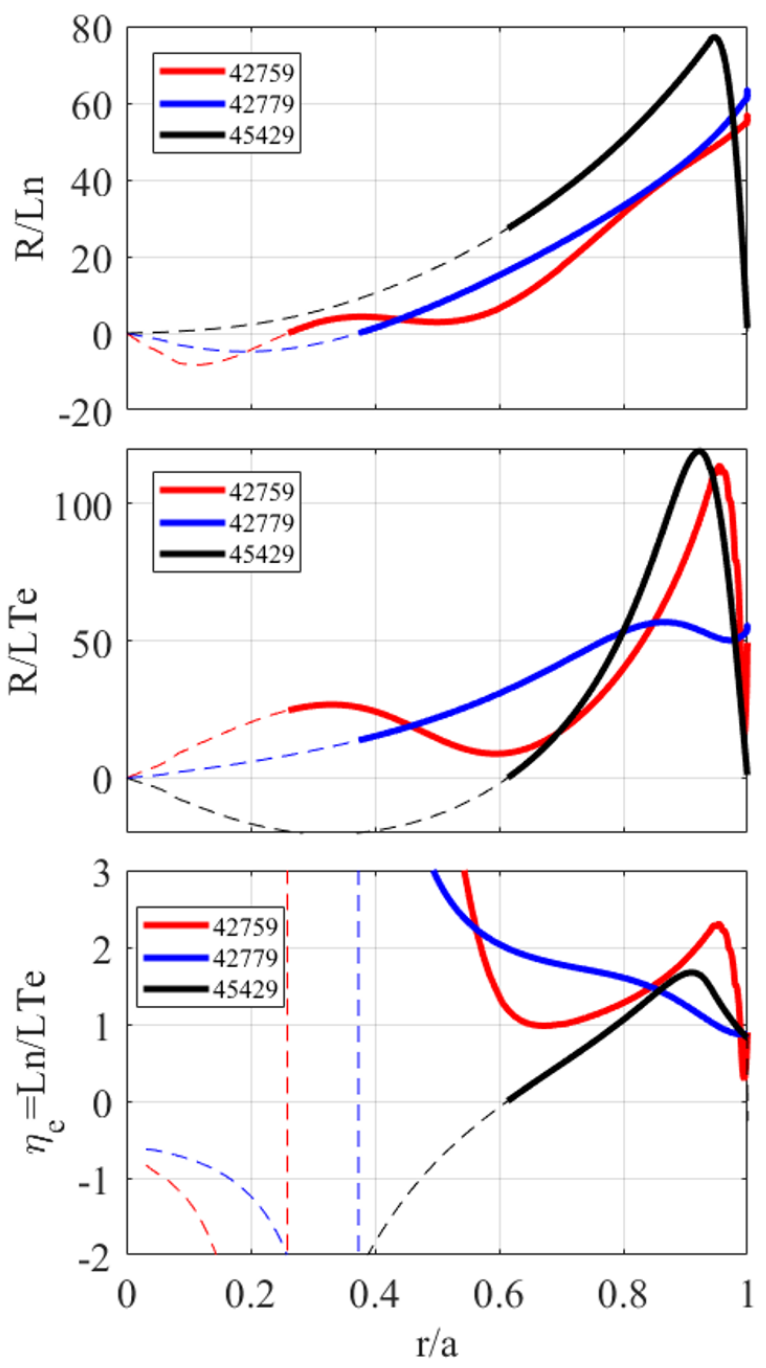

Figure 4. Normalized density (top) and electron temperature gradients (middle) and $\eta_{e}$ parameter (bottom) for the three reference discharges whose profiles are shown in figure 2 Dotted lines are used in the regions where $\partial n / \partial r$ and $\partial T_{e} / \partial r$ have opposite sign and solid lines otherwise.

the location of the maximum of instability, as shown in figure 3 . The maximum instability is concentrated in outer regions $(r / a>0.8)$ in scenarios peaked and hollow, while it moves to $r / a<0.8$ in the broad one (see figure 3). This result is consistent with experimental measurements of density fluctuations by DR.

In figure 5 the density fluctuations spectra measured by the DR system in the peaked and broad scenarios are shown vs the perpendicular wavenumber for several radial regions separately. It is clear from figure 5 that measurements at different radial positions in peaked scenario have clear differences for wavenumbers in the range $4 \mathrm{~cm}^{-1}<\mathrm{k}_{\perp}<10 \mathrm{~cm}^{-1}$ : the spectral amplitude is larger for outer positions, $r / a>0.75$. Note the $\log$ scale in the figure. In the broad case, however, there are not significant 
differences between measurements at different radial positions within the radial range of measurement. Note that the range of measurement of the DR system slightly changes from peaked to broad scenario, as it depends on the density profile, which has slightly smaller densities in the plasma edge for the broad scenario (see figure 2).

The radial location of maximum instability found in simulations is very similar in the peaked and hollow scenarios, as shown in figure 3 , and the wavenumber spectra are also similar in both cases, with slight differences. The wavenumber of maximum instability (growth rate) in the peaked scenario is slightly smaller than that in the hollow one $(m=148$ versus $m=116)$. The most unstable mode has a larger growth rate in the hollow case $\left(\gamma=7.7 \cdot 10^{5} \mathrm{~s}^{-1}\right)$ than that in the peaked one $\left(\gamma=6.8 \cdot 10^{5} \mathrm{~s}^{-1}\right)$ as shown in table 1 . Furthermore, there is a larger contribution of modes with $k_{\theta}=7-9 \mathrm{~cm}^{-1}$ in the peaked scenario than in the hollow one. These details are consistent with the experimental observations in wavenumber spectra for these two scenarios, which shows larger amplitude for $k_{\perp}=7-10 \mathrm{~cm}^{-1}$ in the peaked scenario while the amplitude at $k_{\perp}<6 \mathrm{~cm}^{-1}$ is larger in the hollow one (see figure 6). Note that the DR system cannot provide absolute values of fluctuation levels [39] due to the lack of an absolute calibration. However, the different channels in the DR system at TJ-II are calibrated, and then a direct comparison of spectral amplitudes in different scenarios is possible.

Note that the quantities $L_{n}, L_{T_{i}}$ and $L_{T_{e}}$, derived from the profiles shown in Figure 2, have significant uncertainties. Taking into account these uncertainties by means of a set of simulations with profiles varying within uncertainties, would require a prohibitively large amount of computational resources.

\subsection{Identification of the instability drive}

Comparing the location of maximum instability found in simulations and the density and $T_{e}$ scale lengths in figure 4 we can try to identify the driving force for the modes found as linearly unstable. With this purpose we analyze simulations without including $E_{r}$ or collisions, in order to account only for the influence of the profile gradients in the destabilization of modes. In figure 7 the spectral amplitude (normalized to its maximum) at $\phi=0$ is shown versus the poloidal mode number and the radial position for a set of simulations for the reference discharges without including $E_{r}$ or collisions. Note that the radial location of maximum instability is very similar in these cases and the corresponding ones shown in figure 3. The growth rates and the mode numbers of the most unstable modes in these ideal cases are larger, however, as shown in table 1.
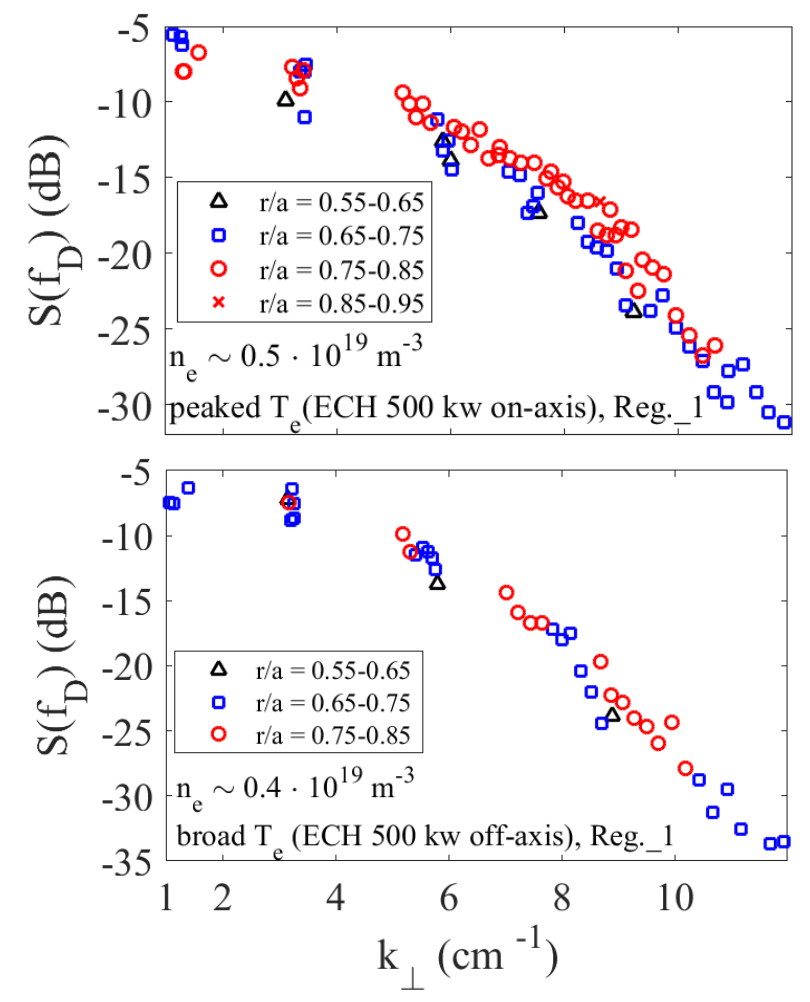

Figure 5. Density fluctuations spectra from the DR system for peaked (top) and broad (bottom) scenarios. Different symbols (and colors) indicate different radial position of measurement. Both cases correspond to measurements in the Region_1 shown in figure 1

From the inspection of Figs. 4 and 7 and Table 1 we can extract some information that can help us to identify the driving force:

- Larger maximum growth rates are found in both peaked $\left(\gamma_{\max }=8.5 \cdot 10^{-5} \mathrm{~s}^{-1}\right)$ and hollow $\left(\gamma_{\max }=9.1 \cdot 10^{-5} \mathrm{~s}^{-1}\right)$ scenarios than in the broad one $\left(\gamma_{\max }=7.4 \cdot 10^{-5} \mathrm{~s}^{-1}\right)$, while $R / L_{n P} \lesssim$ $R / L_{n B}<<R / L_{n H}$ for $r / a>0.5 \S$.

- In the outer region of the plasma $(0.8<r / a<$ $0.95)$, where the maximum instability is found for peaked and hollow scenarios, it happens that both $R / L_{T_{e} P}>>R / L_{T_{e} B}$ and $R / L_{T_{e} H}>>R / L_{T_{e} B}$.

- As compared to peaked and hollow scenarios, in the broad one the maximum instability moves to inner positions $(0.6<r / a<0.8)$, where both $R / L_{T_{e} B}>R / L_{T_{e} P}$ and $R / L_{T_{e} B}>R / L_{T_{e} H}$.

where we have used the subindex $\mathrm{P}, \mathrm{B}$ and $\mathrm{H}$ to label peaked, broad and hollow scenarios, respectively.

From these considerations, we can preliminarily conclude that these modes are primarily driven by the electron temperature gradient rather than by the

$\S$ Note that these values, corresponding to simulations without $E_{r}$ or collisions, are larger than those in Section 4 

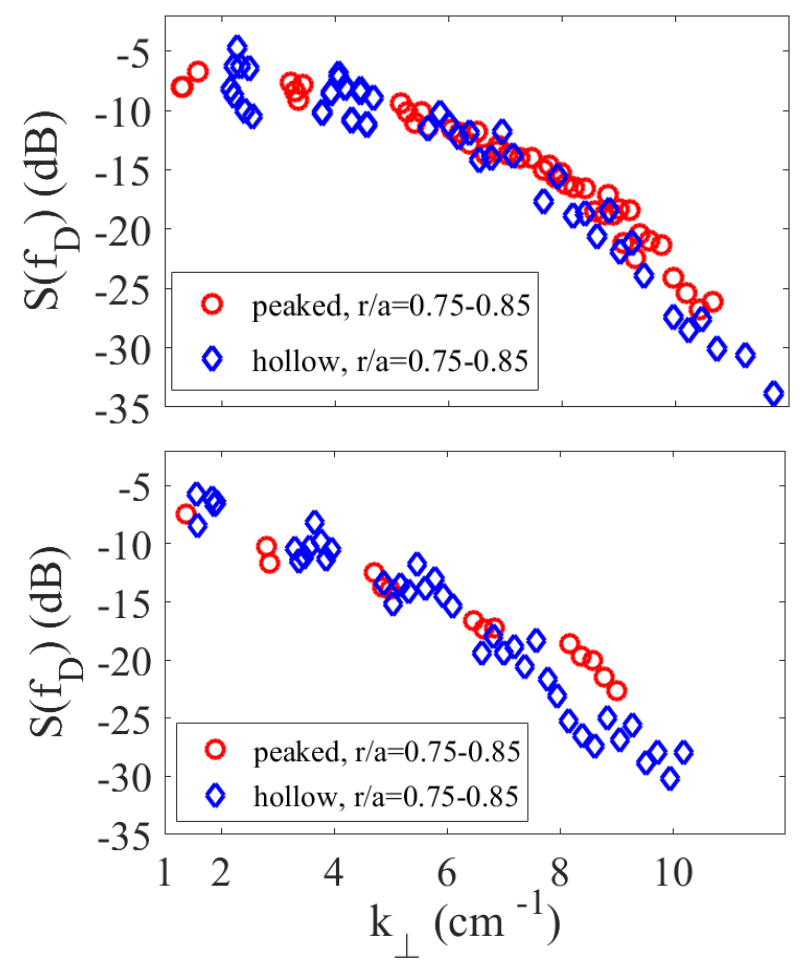

Figure 6. Density fluctuations spectra from the DR system for peaked and hollow scenarios measured at $r / a=0.75-0.85$ in Region_1 (top) and Region_2 (bottom).

density gradient. This will be also concluded in the sensitivity analysis presented in section 4.2 .2 .

4.2.1. Wave-particle interaction analysis. Following 56] we can analyze the wave-particle interaction by relating the growth rate with the evolution of electrostatic $\left(E_{f a}\right)$ and kinetic $\left(E_{k a}\right)$ energies:

$$
E_{f a}=\frac{q_{a}}{2} \int\left(\left\{n_{a}\right\}-n_{a}\right) \varphi d \mathbf{x}
$$

and

$$
E_{k a}=\int\left(m_{a} \mu B+\frac{v_{a \|}}{2}\right) f_{a} d \mathbf{R} \mathrm{d} \mathbf{v},
$$

where $\int \ldots d \mathbf{x}$ means integration in the full volume.

It can be demonstrated [57, that

$\gamma=\frac{1}{2 E_{\mathrm{fa}}} \frac{\mathrm{dE}_{\mathrm{fa}}}{\mathrm{dt}}=\frac{-1}{2 E_{\mathrm{fa}}} \frac{\mathrm{dE}_{\mathrm{ka}}}{\mathrm{dt}}=\frac{-q_{a}}{2 E_{\mathrm{fa}}} \int \delta f_{a} \frac{\mathrm{d} \mathbf{R}_{\mathrm{a}}}{\mathrm{dt}} \cdot \nabla\langle\varphi\rangle \mathrm{d} \mathbf{R} \mathrm{d} \mathbf{v}$.

Plugging in this equation the evolution equation $\dot{\mathbf{R}}$ given by Equation (2) we can separate the contribution of the different terms in $\dot{\mathbf{R}}$ to the evolution of the electrostatic field energy and then the different contributions to the total growth rate of each kinetic species as

$$
\gamma_{a}=\gamma_{v_{\|} a}+\gamma_{\nabla B a}+\gamma_{\kappa a}+\gamma_{\nabla P a},
$$

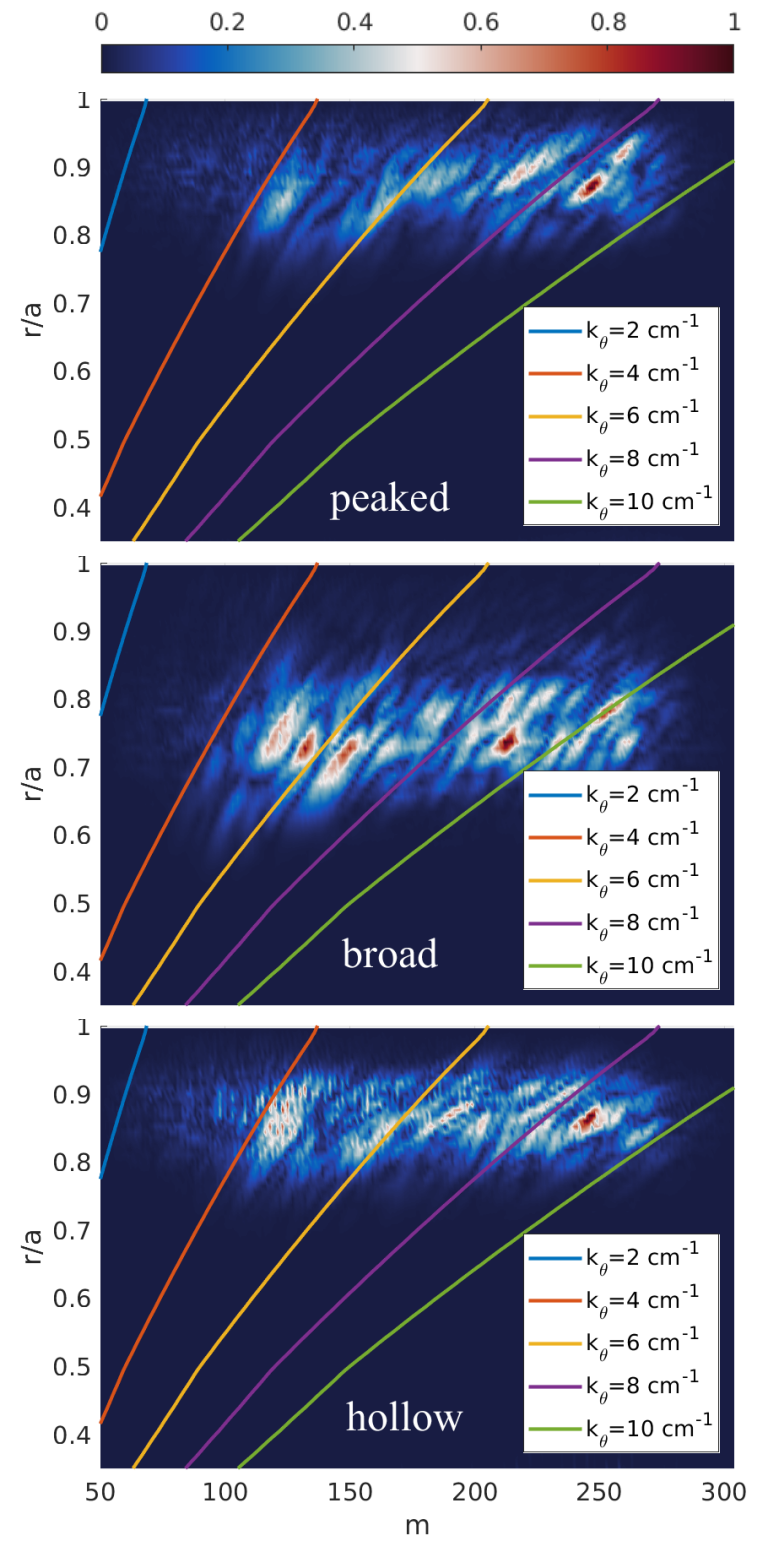

Figure 7. Spectral amplitude of the perturbed potential (normalized to its maximum value) in a set of linear simulations using experimental density and temperature profiles (figure 2) without including $E_{r}$ or collisions.

with

$\gamma_{v_{\| a}}=\frac{-q_{a}}{2 E_{\mathrm{f}}} \int v_{a \| \mid} \mathbf{b} \cdot \nabla\langle\varphi\rangle \delta f_{a} \mathrm{~d} \mathbf{R} \mathrm{d} \mathbf{v}$,

$\gamma_{\nabla B a}=\frac{-q_{a}}{2 E_{\mathrm{f}}} \int \frac{\mu_{a} B(\mathbf{b} \times \nabla B)}{\left(B^{*} \Omega_{a}\right)} \cdot \nabla\langle\varphi\rangle \delta f_{a} \mathrm{~d} \mathbf{R} \mathrm{d} \mathbf{v}$,

$\gamma_{\kappa \mathrm{a}}=\frac{-q_{a}}{2 E_{\mathrm{f}}} \int \frac{v_{a||}^{2}(\mathbf{b} \times \nabla B)}{\left(B^{*} \Omega_{a}\right)} \cdot \nabla\langle\varphi\rangle \delta f_{a} \mathrm{~d} \mathbf{R} \mathrm{d} \mathbf{v}$,

$\gamma_{\nabla P a}=\frac{-q_{a}}{2 E_{\mathrm{f}}} \int \frac{v_{a \| \mid}^{2}(\nabla \times B)_{\perp}}{\left(B^{*} \Omega_{a}\right)} \cdot \nabla\langle\varphi\rangle \delta f_{a} \mathrm{~d} \mathbf{R} \mathrm{d} \mathbf{v}$.

This is known as the $\mathbf{j} \cdot \mathbf{E}$ analysis, as $\mathbf{j}=q_{a} \frac{\mathrm{d} \mathbf{R}_{\mathbf{a}}}{\mathrm{dt}}$ defines a current whose correlation with the electric 

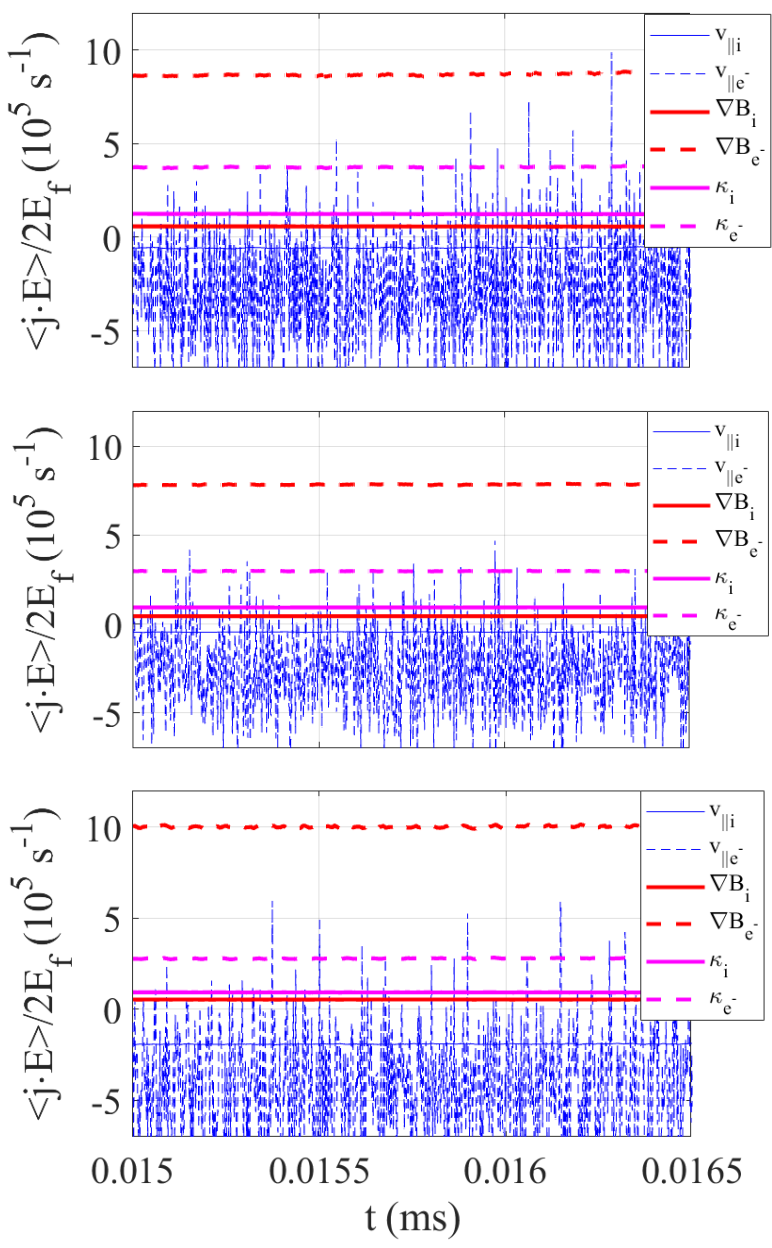

Figure 8. Contribution of the different elements from the particle dynamics to the growth rate of the modes as defined in Equations 10 to 13 for the peaked (top), broad (middle) and hollow (bottom) cases. Positive sign corresponds to destabilization of the modes and negative to a stabilizing effect.

field $\mathbf{E}=-\nabla\langle\varphi\rangle$ gives the rate of energy interchange between particles and fields. It allows distinguishing if the unstable modes are driven either by ions or by electrons and permits us to compare the relative influence of the different elements of the particle dynamics to the mode destabilization/stabilization. According to the definitions in Equations (10) to (13), a positive sign means that the corresponding term contributes to destabilizing the waves (energy going from particles to the wave) and a negative sign corresponds to a stabilizing effect (electrostatic energy converted into kinetic energy of particles).

Figure 8 shows the evolution in time of the different contributions to the growth rate defined in Equations 10p-13 for the three plasma scenarios studied. According to the $\mathbf{j} \cdot \mathbf{E}$ analysis, these modes are found to be driven mainly by electrons while there is a very small net contribution of ions to the destabilization. The parallel dynamics both from ions and from electrons is stabilizing (see figure 8). Note that the component associated to the parallel velocity of electrons is much noisier than any other in the plot, probably due to the fast parallel electron dynamics as compared to the evolution of the electric field.

The largest contribution to the growth of these modes is $\gamma_{\nabla B_{e}}$ from electrons, which is a signature of the trapped electron character of the modes. There is an important contribution also coming from the curvature, $\gamma_{\kappa_{e}}$. The contributions $\gamma_{\nabla P i}$ and $\gamma_{\nabla P_{e}}$ of ions and electrons (not shown in the figure) are negligible in all these cases, as corresponding to the MHD equilibrium used. A vacuum equilibrium is used in all the simulations as the experimental plasmas have very small $\beta\left(\sim 10^{-3}\right)$, but this results do not change significantly when the simulation is carried out with an MHD equilibrium including the experimental plasma pressure profile in the VMEC calculation, which confirms that a vacuum equilibrium is a good approximation, as it was also found in [54.

The kind of modes found unstable is very similar in the three plasma scenarios, with very similar relative contributions to the growth rate as shown in figure 8 .

The type of electron-driven modes found is not surprising as previous studies have shown that in TJ-II modes driven by the ion temperature gradient (ITGs) are not unstable under typical plasma conditions [55. This analysis confirms also that in these plasma scenarios the ITG modes are also stable with kinetic electrons.

In this analysis, the simulations without electric field or collisions, shown in figure 7 were studied with the intention of isolating the character of modes that depend on the profiles alone. Including the neoclassical electric field and collisions in the simulations as in the cases shown in figure 3 has a stabilizing effect, thus reducing the growth rate with respect to the case without $E_{r}$ (see Table 1), but without changing the picture of wave-particle interaction. The relative contribution of the different terms in Equations (13) to 13) are very similar in both cases.

In simulations including the background electric field but not the collisions (not shown here) the spectrum of unstable modes is modified with respect to the cases without collisions nor electric field, the amplitude of large- $m\left(k_{\theta}>8 \mathrm{~cm}^{-1}\right)$ modes being significantly reduced. The growth rate is also significantly reduced. When collisions are also included in the simulation, the growth rate is further reduced. This result indicates the stabilizing influence of both the collisions and the electric field on these modes. The stabilizing effect over large- $m$ modes, which is mainly due to the sheared electric field rather than to collisions, can be appreciated by comparing figures 3 and 7 . 


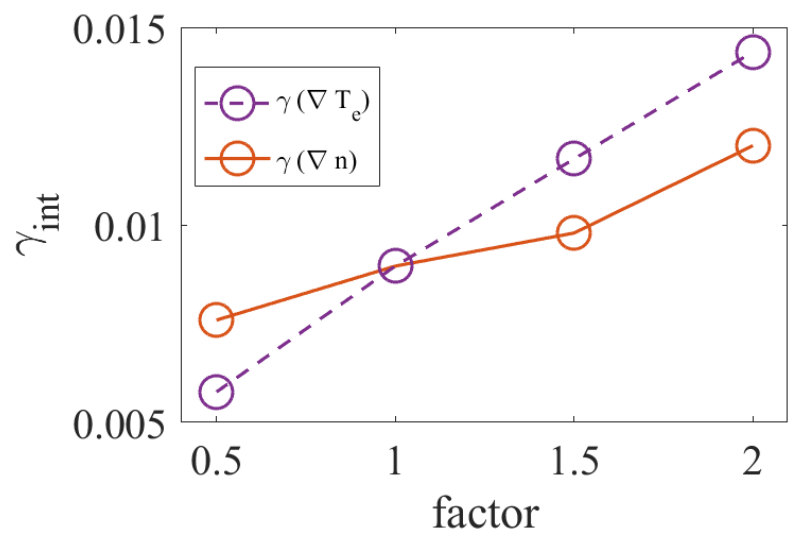

Figure 9. Growth rate (obtained from the volume-integrated field energy) for a set of linear simulations in which the density and temperature gradients are scaled by a factor ranging from 0.5 to 2 .

4.2.2. Sensitivity to gradients. Trapped electron modes (TEM) can be destabilized either by the density gradient [6] or by the temperature gradient [58]. As explained in Section 4.2 the driving force for the modes found unstable appears to be the electron temperature gradient. In order to better clarify this point, the sensitivity to the density and temperature gradients has been studied in a set of simulations in which the experimental density and temperature profiles from peaked scenario (see figure 2) are used while their gradients are modified.

The density and temperature profiles are introduced in EUTERPE by providing both the values of the ion and electron density and temperature and their gradients for a set of radial positions from $r / a=0$ to $r / a=1$. Providing the profiles and their gradients is redundant, but allows the flexibility to study separately the dependency with both profiles values and their gradients. Note that this can lead to physically inconsistent pairs of density/temperature and their gradient, however this option is still useful for testing purposes, as in this case.

In this test, either the density or the temperature normalized gradient is modified by applying a scale factor ranging from 0.5 to 2 and a linear simulation is run for each set of profiles, without including the electric field or accounting for the collisions. The results of this sensitivity study are shown in figure 9 , where the integrated growth rate, $\gamma_{\text {int }} \mathbb{\|}$. is shown versus the scale factor applied to the density and temperature normalized gradients. It is clear from the figure that the growth rate is more sensitive

II A global growth rate can be obtained by fitting the total, volume-integrated, electrostatic energy to an exponential. This growth rate is dominated by the most unstable modes and close to, although not exactly the same as, their growth rate. to the electron temperature gradient than to the density gradient, which is interpreted as a proof that these modes are primarily destabilized by the electron temperature gradient, rather than by the density gradient as it is the case of standard TEM, thus confirming the initial conclusion obtained at the beginning of section 4.2 Both the density and the electron temperature gradients are destabilizing, however.

We can distinguish two kinds of modes that are promoted either by the density gradient or by the electron temperature one. The relative importance of modes in the lower region of the Fourier spectrum, with $m<150$, decreases when the electron temperature gradient is increased, while modes with $m>200$ are favored. The density gradient has basically the opposite effect: increasing it reduces the relative contribution of large-m $(m>200)$ modes below that of the small-m modes. In all these simulations the contribution of ions to the growth rate is negligible as compared to that of electrons.

We could identify the lower-m modes as more $\nabla n$ driven and with a larger ion contribution, while the larger-m modes would be more $\nabla T_{e}$-driven and with a larger contribution of electrons to their destabilization. In addition, the lower-m modes which are promoted by a larger density gradient show a peak amplitude in regions of local minima of the magnetic field strength, as could be expected for TEM.

\subsection{Phase velocity}

The measurement of the electric field by Doppler reflectometry is based on the assumption that the poloidal rotation of the fluctuations is dominated by an $\mathbf{E} \times \mathbf{B}$ global flow with advects the density fluctuations to which the system is sensitive. The electric field is then obtained from the Doppler shift of the density fluctuations spectrum. The assumption that the flow is dominated by $\mathbf{E} \times \mathbf{B}$ and the measured electric field is correct has been proved to be the case in some conditions in TJ-II [59]. However, in some situations, such as when the electric field is very small, the poloidal rotation of fluctuations can be dominated not by the $\mathbf{E} \times \mathbf{B}$ but by the natural phase velocity at which the unstable modes propagate instead. This propagation can even change sign when different modes are excited, for instance in the transition from ITG (propagating in the ion diamagnetic direction) to TEM (in the electron diamagnetic direction) turbulence [60, or in presence of ubiquitous modes, whose direction of propagation can change with plasma conditions [6, 61. As the velocity of propagation of modes in the simulation can be calculated from the wavenumber spectrum, this property is susceptible of comparison between simulations and experiments. 

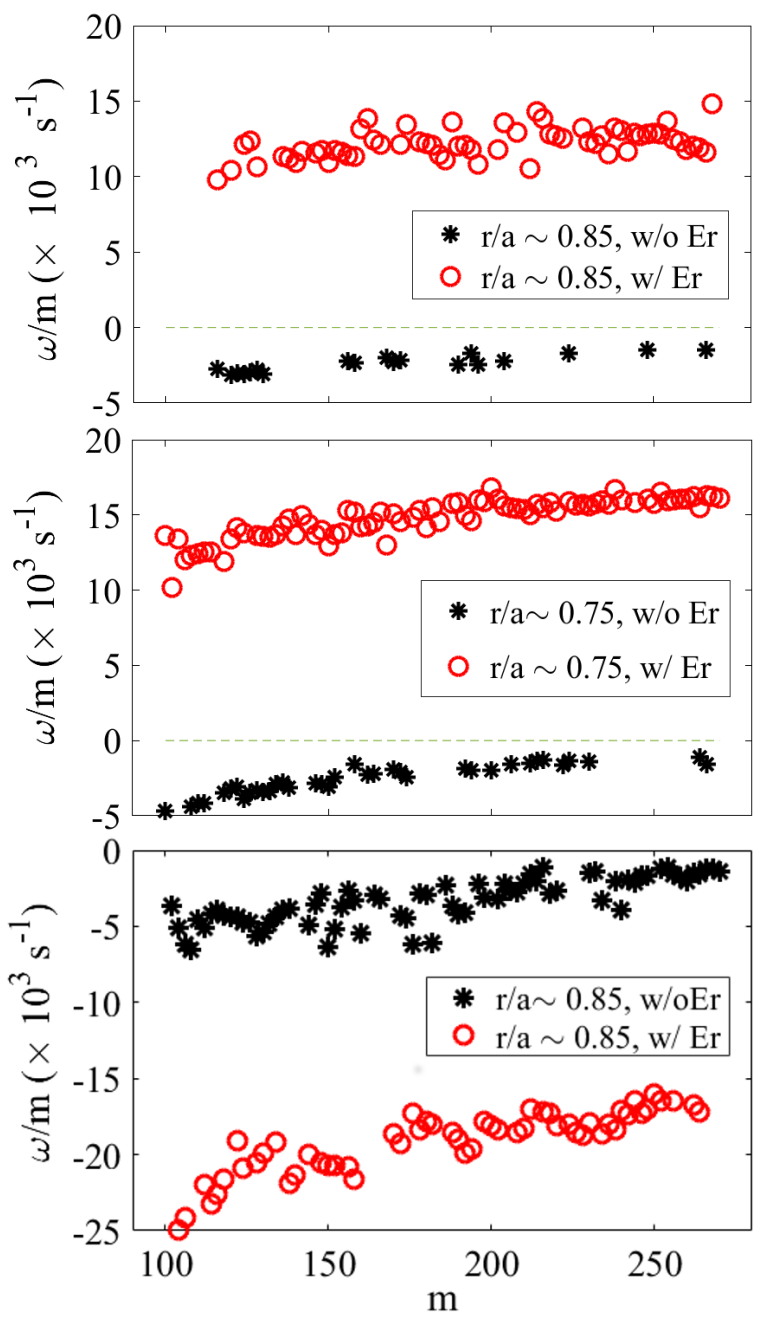

Figure 10. $\omega / m$ (proportional to the phase velocity of fluctuations, $\left.v_{\theta}=\omega /(m \nabla \theta)\right)$ obtained from linear simulations for the reference discharges (\#42759, \#42779 and \#45429) at the radial positions $r / a=0.85,0.75,0.85$, respectively. Both the case without ambipolar electric field and also the case including the electric field are shown. A positive sign means propagation in the ion diamagnetic drift direction.

In figure 10 the quantity $\omega / m$, with $\omega$ being the frequency of the mode is shown versus the mode number, $m$, for a set of linear simulations using the experimental profiles of the reference discharges shown in figure 2 at specific radial positions close to the location of maximum instability in each case. Note that this quantity is related to the phase velocity, $v_{\theta}=\omega / k_{\theta}=\omega /(m|\nabla \theta|)$, with $|\nabla \theta|$ depending on the local position. For the region of measurements of the DR system in the experiments carried out in the standard configuration, it is in the range $|\nabla \theta| \sim 3-7$. Positive sign in figure 10 means propagation in the ion diamagnetic drift direction. Both the cases without ambipolar electric field or collisions (whose spectra are shown in figure 7 ) and also the simulations including the electric field and collisions (whose spectra are in figure 3 are shown in this figure. In the simulations without electric field the phase velocity of the modes is going in the electron drift direction, as would be expected for TEM modes. It has to be noted that $\omega$ in this plot is obtained from the poloidal wavenumber spectra at $\phi=0 \mathbb{9}$, shown in figures 3 and 7 with a fit of the time evolution of the spectrum amplitude at a specific mode number to a model of growing sinusoidal oscillations. In all cases the phase velocity has a dependency on the wavenumber, increasing with $m$. The change in $\omega / m$ as $m$ increases is slightly smaller for peaked scenario than for the broad and hollow ones.

The effect of the ambipolar electric field on the phase velocity of the modes, which is shifted in the presence of an $\mathbf{E} \times \mathbf{B}$ rotation, is clearly seen in figure 10. The ambipolar electric field is positive in the region of DR measurement in both the peaked $\left(E_{r} \simeq 2.5 \mathrm{kV} / \mathrm{m}\right.$ at $\left.r / a=0.85\right)$ and broad $\left(E_{r} \simeq\right.$ $3.5 \mathrm{kV} / \mathrm{m}$ at $r / a=0.75)$ scenarios, while in the third scenario it is negative $\left(E_{r} \simeq-3 \mathrm{kV} / \mathrm{m}\right.$ at $\left.r / a=0.85\right)$. The direction of propagation of modes in the peaked and broad scenarios in the simulations including the $E_{r}$ is reversed to go in the ion diamagnetic direction with respect to the case without $E_{r}$, due to the influence of the electric field. In the third scenario, the negative background electric field increases the velocity of propagation of modes in the electron drift direction. Note that the effect of the electric field is not only the Doppler shift in frequency/velocity. Its shear also has a stabilizing effect, mainly on large-m modes, as explained in section 4.2.1.

In figure 11 the perpendicular velocity as deduced from the DR measurements in peaked, broad and hollow scenarios vs the wavenumber is shown for comparison with simulations. As the measurements at different radial positions can be affected by different $E_{r}$, and consequently different Doppler shifts, the velocities for different radial positions are shown separately to make clearer the dependence with the wavenumber. Only measurements at the Region_2 are shown in this figure for clarity. If the assumption $v_{p h}<<V_{E x B}$ is correct, then $v_{\perp}$ should not depend on $k_{\perp}$, and this is the case in the peaked scenario, in which the experimental $v_{\perp}$ show not clear dependence with $k_{\perp}$, in rough agreement with the smaller dependence of $\omega / m$ with $m$ found in simulations.

A strong dependence of the perpendicular velocity of propagation of density fluctuations from DR measurements with the wavenumber is observed in the hollow scenario (see figure 11 and 39 for details), which is in qualitative agreement with results from simulations previously presented. The experimental

9 The diagnostic of wavenumber spectra presently implemented in EUTERPE is limited to $\phi=0$ for the poloidal spectrum and to $\theta=0$ for the toroidal modes. Work is in progress to extend these diagnostics. 

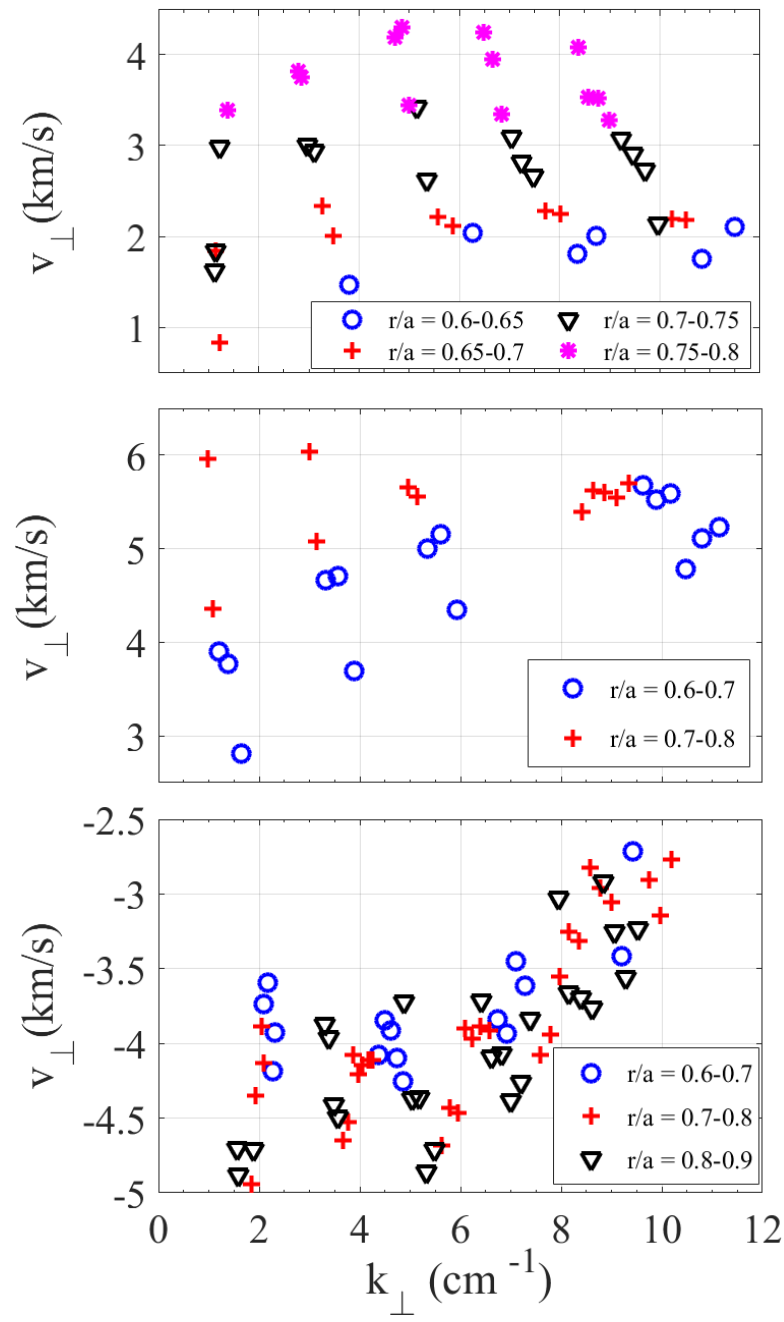

Figure 11. Perpendicular velocity from the DR measurements vs the perpendicular wavenumber for peaked, broad and hollow scenarios.

propagation velocities go in the electron diamagnetic direction and the speed reduces with increasing wavenumbers, from $u_{\perp} \sim 5 \mathrm{~km} / \mathrm{s}$ to $u_{\perp} \sim 2.5 \mathrm{~km} / \mathrm{s}$ in the range $k_{\perp}=3.5-12 \mathrm{~cm}^{-1}$ (see figure 11). In the broad scenario the dependency of the perpendicular velocity with the wavenumber was not as evident as for the hollow one. However, a slight dependence with $k_{\perp}$ appears also in this case when the experimental data are plotted for different radial positions separately (see figure 11.

A qualitative agreement is found between the phase velocity dependency with $k$ found in simulations and that of the experiments, particularly in the hollow scenario. In both experiment and simulation $v_{\perp}$ (or $\omega / m$ ) increases with the wavenumber, which indicates that the phase velocity should be taken into account to calculate the electric field from DR measurements in this case. Note that a quantitative agreement can not be expected. First, the spectra from simulations are obtained at $\phi=0$ and not at the plane of measurement of the DR system. In addition, there is a dependency on $\nabla \theta$ in the phase velocity, with $\nabla \theta$ varying with the position, which is not included in the quantity $\omega / m$. In order to make a quantitative comparison, the spectrum should be diagnosed at the plane of measurement of the DR system and the local values of $\nabla \theta$ taken into account in the calculation of $v_{\perp}$ from simulations data. Work is in progress to improve the diagnostics from simulations and the comparison between simulations and experiment in this respect. In addition, the simulations have been carried out including a model electric field, which can have a significant uncertainty and does not include variations of the electric field along the flux surface, which are known to be relevant in some plasma regimes 63. Furthermore, the phase velocity of fluctuations could also be modified in nonlinearly saturated simulations.

\subsection{Influence of the ion mass}

The influence of the ion mass on the fluctuations has been studied both experimental and numerically. To this end, the plasma peaked scenario has been reproduced with both Hydrogen and Deuterium as base gas obtaining very similar density, temperature and electric field profiles in both cases. Simulations have been carried out also with $\mathrm{H}$ and $\mathrm{D}$ ions, using the same profiles from figure 2 for the shot \#42759 as input and including $E_{r}$ and collisions.

In figure 12 the normalized amplitude of the potential spectrum is shown versus the normalized radius, $r / a$, and poloidal mode number for these simulations. Note that the wavenumber range in these simulations is not exactly the same as in those of Fig 3). The spectra show no relevant differences, as expected for electron-driven modes (TEMs), which can be expected to be only slightly affected by the ion dynamics. The growth rate of unstable modes is smaller with $\mathrm{D}\left(\gamma_{\max }=4.7 \cdot 10^{5} \mathrm{~s}^{-1}\right)$ than with $\mathrm{H}$ $\left(\gamma_{\max }=6.8 \cdot 10^{5} \mathrm{~s}^{-1}\right)$, but the spectrum of linearly unstable modes is very similar in both cases. For ion-driven modes (ITG) the wavenumber of unstable modes would reduce by a factor $\sqrt{2}$ for D as compared to the case with $\mathrm{H}$, and a change in this direction would be expected if the ions played a key role in driving these modes. This result is in agreement with the density fluctuations measurements by DR, which show very similar density fluctuations spectra in both cases also (see 65) and both support the conclusion that the relevant unstable modes in these plasmas are driven by electrons.

The low dependence of turbulent DR spectra (and simulations) with the ion mass contrasts with results reported for LHD [66], showing a significant isotope effect on fluctuation levels, turbulent fluxes and the 


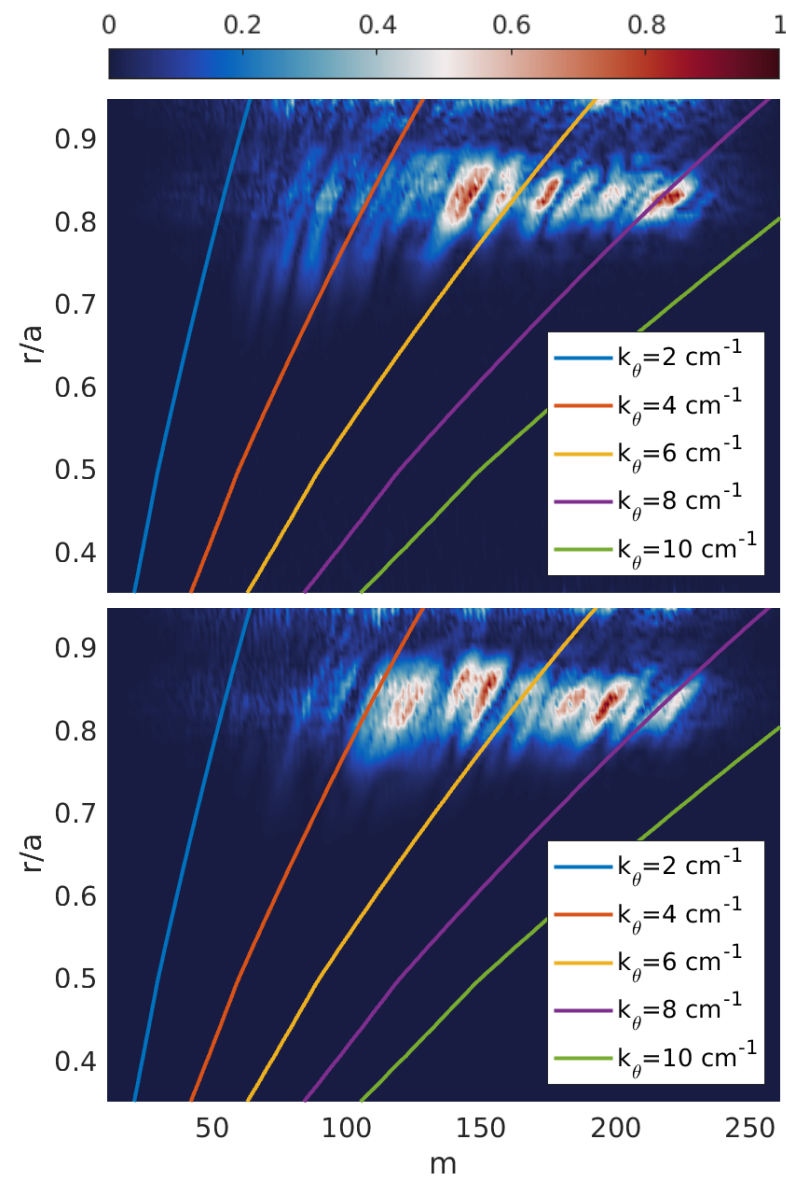

Figure 12. Normalized spectral amplitude of potential in two simulations with $n, T_{e, i}$ (figure 2 and $E_{r}$ experimental profiles from discharge \#42759 and $D$ (top) and $H$ (bottom).

energy transfer to zonal flows in TEM turbulence. This could point to different roles of the zonal flows in the non-linear saturation of turbulence in TJ-II and LHD. Actually, it is already known that the linear response of zonal flows in both devices is quite different [22].

\subsection{Localization of modes on the flux surface}

Electrostatic unstable modes in TJ-II are found to reach their maximum amplitude in locations of bad magnetic field line curvature and small local magnetic shear [55, 9], as in other stellarators [7, 8, 10]. In figure 13 the amplitude of the potential is shown versus the poloidal $(\theta)$ and toroidal $(\phi)$ magnetic coordinates angles in a radial location close to the maximum instability for the set of simulations from figure 3 . It is clear from the figure that the instability has the maximum amplitude along a narrow stripe close to the field line crossing at $\theta=0, \phi=0$, which is shown in the figure with a green line.

In figure 14, the magnetic field strength is shown versus poloidal and toroidal magnetic coordinate

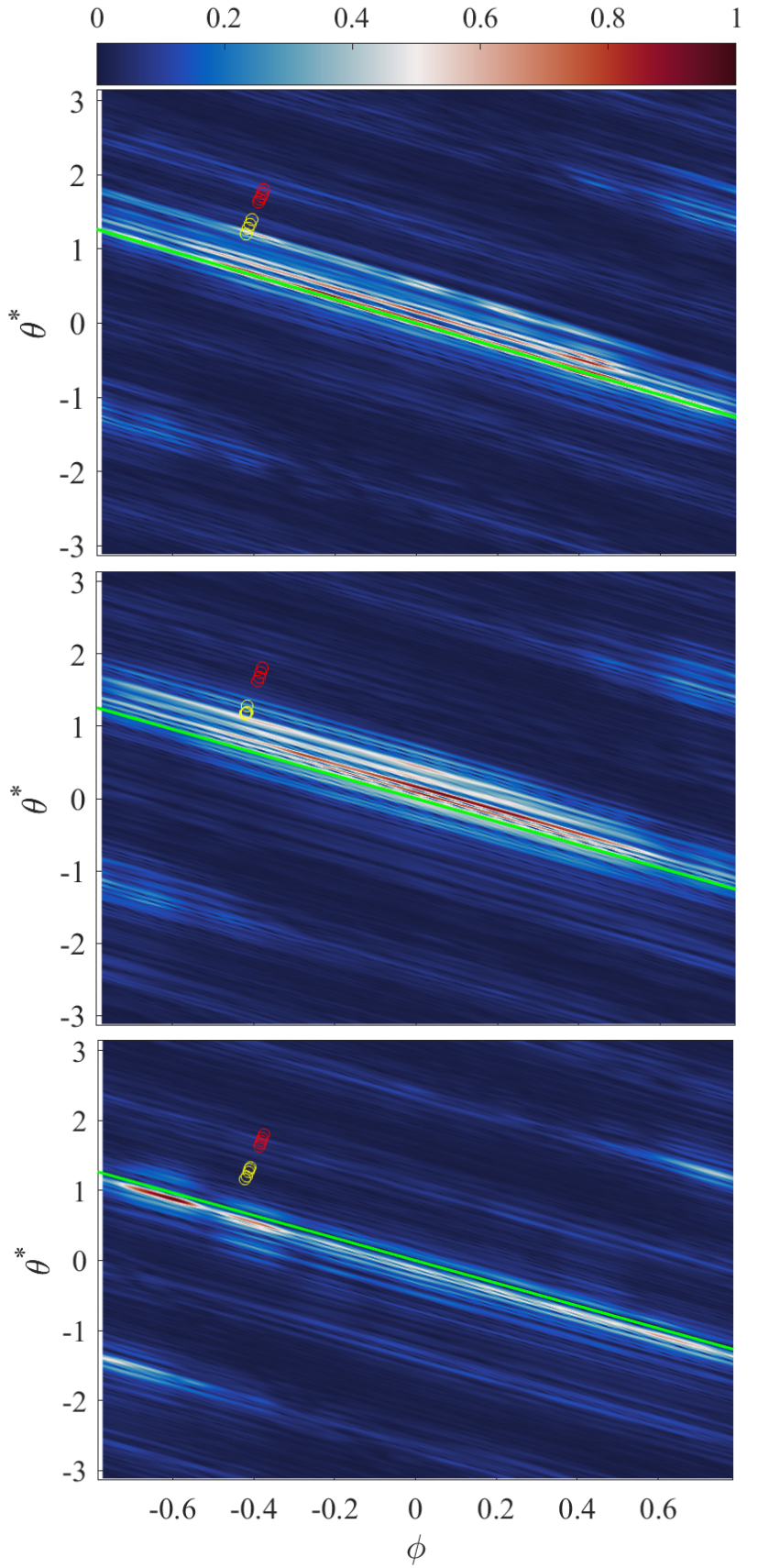

Figure 13. Amplitude of the potential (normalized to the maximum value) in the linear simulations (shown in figure 3, carried out int he standard configuration for the three plasma scenarios, represented versus poloidal and toroidal angles in a flux surface close to the radial location of maximum instability in each case $(r / a \simeq 0.85,0.75$ and 0.85 , respectively). The positions of DR measurements over this flux surface in Region_1 and Region_2 are shown with red and yellow circles, respectively. 


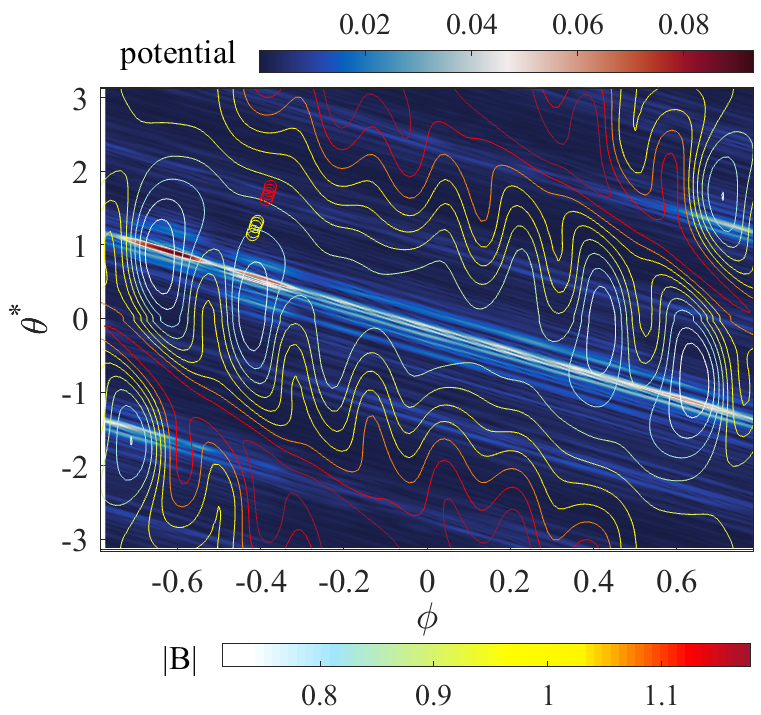

Figure 14. Normalized amplitude of potential versus poloidal $(\theta)$ and toroidal $(\phi)$ angles in a flux surface at $r / a \simeq 0.85$ (same as Figure 13-bottom) compared to the magnetic field strength, represented with contours. The positions of DR measurements over this flux surface in Region_1 and Region_2 are shown with red and yellow circles, respectively.

angles at the same radial position, $r / a \sim 0.85$, together with the potential shown in figure 13 bottom. It can be noticed that in the hollow case, the maximum instability is located near the minima of the magnetic field strength. This is consistent with the fact that hollow scenario has a larger density gradient at the edge region (see figure 4) and we observed in the sensitivity analysis presented in section 4.2 .2 that when the density gradient is increased, the modes have a smaller wavenumber and are located at regions of minimum magnetic field strength.

The strong localization of the regions of maximum instability of electrostatic modes previously found in simulations motivated the measurement at two poloidally separated locations with the DR system (Region_1 and Region_2) in all these experiments, as shown in figure 1. A systematic difference in the amplitude of the density fluctuations spectra in both regions has been found in some regimes, in particular in the first scenario. The $\tilde{n}$ spectra measured in peaked scenario at the Region_1 has clearly larger amplitude than that measured at Region_2 as shown in figure 1 . bottom (see also 39] for details).

In figure 15 top the normalized amplitude of the potential in a linear simulation carried out in the standard magnetic configuration with the profiles from shot \#42759 (peaked) is shown in the plane of measurement of the DR system. In the same figure, the positions of measurements of the DR system at the two regions are shown with circles. The red circles correspond to Region_1 and the black ones to
Region_2. A higher amplitude of density fluctuations is found in Region_1, as shown in figure 1 1 and then, the discrepancy with simulation results is clear.

The sensitivity of the spatial localization to the neoclassical electric field has been studied in a set of simulations keeping the same density and temperature profiles and changing electric field profile. While the electric field has been shown to displace poloidally (and also radially) the location of maximum instability of ITG modes 13, 14, for these electron-driven modes the displacement due to the electric field is very small and the maximum instability is always found at similar poloidal locations on the flux surface. Simulations without including the electric field or including it scaled by a factor 2 show the maximum amplitude of unstable modes at similar locations.

The poloidal localization of ITG modes in tokamaks has been shown to be affected by the density and/or temperature gradients 62, the maximum of instability being displaced when the gradients are increased, however, for the TEM modes found here the influence of the density and temperature gradients is not relevant, as proven in the different simulations presented in section 4.2.2 Only a change of the position of maximum amplitude towards minimum magnetic field strength regions and within the same "high-amplitude" stripe is found when the density gradient is increased, but the poloidal localization of the "high-amplitude" stripe is very similar.

The poloidal localization of unstable modes over the flux surface appears in simulations as a robust characteristic which is not much affected by electric field, density or temperature gradients that are fluxsurface functions. The localization of instabilities could also be affected by density/potential variations over the flux surface, which are known to be significant under some conditions 63 and can contribute to the electric field with a significant local component. Work is in progress to include this kind of advanced-neoclassical features in the gyrokinetic simulations.

Finally, the non-linear saturation of instabilities could affect the localization of maximum amplitude in fluctuations as compared to the results of linear simulations. Work is also in progress in this direction.

\subsection{Influence of the rotational transform $t$}

The unstable electrostatic modes in TJ-II are located on narrow stripes aligned with the magnetic field lines [9], as shown in the previous section. As the magnetic field line slope is defined by the rotational transform, the location of maximum instability of these modes is observed to depend on this parameter in simulations.

With the goal of studying the change of location of maximum instability with the rotational transform, the peaked scenario previously described, carried out 

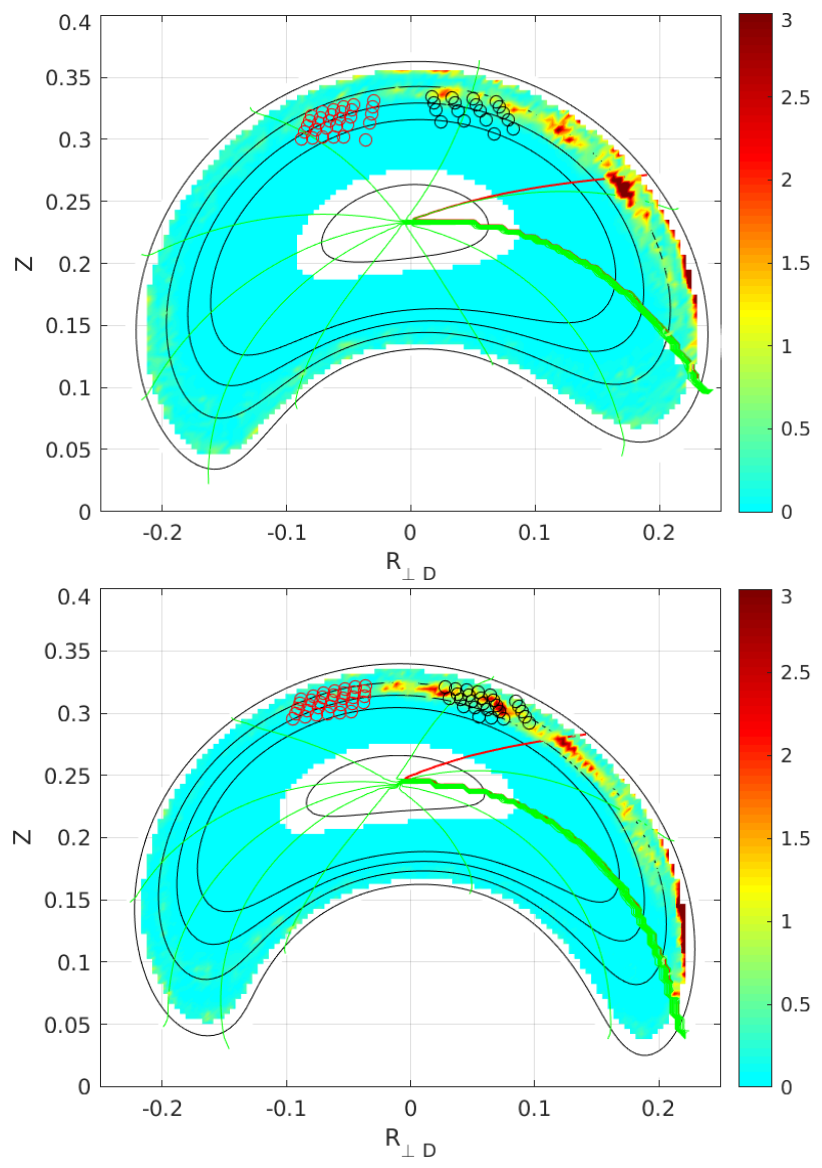

Figure 15. Amplitude of potential in the plane of measurement of DR system for simulations with experimental $n, T_{e, i}$ and $E_{r}$ profiles from shot \#42759 in the standard magnetic configuration (top) and the 42_100_69 one (bottom). The location of DR measurements is shown with circle sets corresponding to a different mirror angle each. The red circles correspond to Region_1 and the black ones to Region_2. Contourns at normalized toroidal flux $s=0.25,0.65,0.75,0.85,1$, with $s \simeq$ $(r / a)^{2}$, are shown with black solid lines. The green lines show constant $\theta$ contourns for $\theta=0.2 \pi, 0.4 \pi, \ldots, 2 \pi$, with a thicker line for $\theta=0$. The red line shows the field lines crossing at $\theta=0$ for toroidal angle $\phi=0$ at all radial positions.

first in the standard magnetic configuration $(t=$ $1.55-1.65)$, was repeated in a configuration, 42_100_69, with a larger rotational transform $(t=2.12-2.24)$. Note that as the rotational transform in the 42_100_69 configuration is increased with respect to the standard one, the global magnetic shear is reduced. The influence of the global magnetic shear on the ITGs in TJ-II has been shown to be small as compared to that of the local one 9 .

The measurements of density fluctuations spectra in this series of discharges shows an inversion of the poloidal asymmetry that was found in the discharges carried out in the standard configuration: a larger amplitude of fluctuations was found in Region_2 in the high iota configuration case.

A simulation with the same profiles of peaked scenario (\#42759) was carried out in the 42_100_69 magnetic configuration and the results are shown in figure 15 -bottom, where the amplitude of the potential in the simulation at the plane of measurement of the DR system is shown together with the position of measurement by the DR system. A clear displacement of the location of maximum instability toward higher $\theta$ angles is found in the simulation, getting it closer to the location of DR measurements in Region_2, which is consistent with the experimental measurements that in this case find the larger amplitude of density fluctuations in Region_2 than in Region_1.

We can say that the experiment and the simulations agree on that the location of maximum fluctuations depends on $t$. However, no clear agreement is found on how $t$ affects the instabilities. While a reasonable qualitative agreement is found in the high iota case, in the standard configuration there is a disagreement between experimental measurements and simulations in respect to the localization of instabilities. The reason for the discrepancy in the case of the standard configuration could be the significant potential variation over the flux surface which was found in some plasma regimes 63, 39, which translates into a local component, not necessarily radial, of the electric field derived from those potential variations. The potential variation along the flux surface manifests in a significant difference between the electric field at regions 1 and 2 in simulations [63, 39. In the high iota configuration, the measurements of the electric field at both poloidal regions are closer than in standard configuration and the sign of the asymmetry is also inverted (see [39]). This point is currently under investigation.

\section{SUMMARY AND CONCLUSIONS}

In this work, recent simulations carried out in TJII magnetic configurations with the global gyrokinetic code EUTERPE are compared with experimental measurements from Doppler Reflectometry in dedicated experiments. In the experiments, the power and deposition location of the ECRH heating were changed, thus modifying significantly the density and temperature radial profiles. Three different scenarios were produced. Global linear simulations of electrostatic instabilities have been run using the experimental density, temperature and electric field profiles from plasma discharges representative of the three scenarios.

Electron-driven modes are found unstable in the plasma gradients region, where both the density and temperature gradients are negative. According to the linear stability analysis, these modes have a TEM character. A sensitivity analysis shows that these modes are more sensitive to the electron temperature 
gradient than to the density gradient, which is in agreement with the dependence of the growth rates of these modes with the profiles gradients, and supports the view that these modes are driven by the electron temperature gradient rather than by the density gradient.

The relevant wavenumbers found in simulations are consistent with the range of unstable wavenumbers found in experimental density fluctuations spectra, the most unstable modes in simulations being close to the position of a knee observed in experimental spectra, which can be identified with the wavenumber region of injection of energy in the spectrum.

The radial variation of the amplitude in experimental density fluctuations spectra measured by DR system in different plasma scenarios is consistent with the location of maximum instability found in simulations, which slightly displaces radially from scenario to scenario as the profiles are modified.

No dependency of the experimental density power spectra with the bulk ion mass is observed, in agreement with the simulations for different ion mass that do not show significant differences in the spectrum of unstable modes. This result is consistent with the expectations for the electron-driven modes found in simulations.

A poloidal propagation of the unstable modes with a phase velocity which increases with the mode number is found in the simulations. The phase velocity is affected (Doppler shifted) by the background, neoclassical, electric field giving a net poloidal propagation velocity for the modes which still has a dependence with the wavenumber. The results are in qualitative agreement with experimental results, particularly in the high collisionality regime (with hollow electron temperature profile), in which the perpendicular velocity measured by the DR system shows clear dependence with the wavenumber.

The unstable modes reach the largest amplitude in the flux surface along narrow stripes aligned with the field lines in regions of negative magnetic field line curvature and small local magnetic shear, in agreement with findings in previous works [7, 8, 9, 10. As the stripes of maximum instability are aligned with the magnetic field lines their poloidal location for a given toroidal angle is sensitive to the rotational transform.

A systematic difference is found between the density fluctuation spectra measured by the DR system at poloidally separated positions on the same flux-surface that is compatible with a poloidal localization of the turbulence source as appearing in numerical simulations. This localization is affected by the rotational transform both in simulations and experiments. However, not full agreement between experiments and simulations is found so far. Good qualitative agreement between simulation results and experiments in this respect is found in a high rotational transform configuration, while disagreement is found in the standard configuration. Work is in progress to further investigate the origin of the discrepancies. The influence of the variations of density and potential over the flux surface on the unstable modes is being considered. Non-linear simulations are under way which can provide a saturated wavenumber spectrum directly comparable with experimental measurements.

\section{AKNOWLEDGEMENTS}

This work has been partially funded by the Ministerio de Economía y Competitividad of Spain projects ENE2015-70142-P and FIS2017-88892-P. The authors thankfully acknowledge the computer resources at MareNostrum and the technical support provided by the Barcelona Supercomputing Center. This work has been carried out within the framework of the EUROfusion Consortium and has received funding from the Euratom research and training programme 2014-2018 under grant agreement No 633053. The views and opinions expressed herein do not necessarily reflect those of the European Commission.

\section{References}

[1] Catto, P. J. 1978 Plasma Phys. 20719

[2] Frieman, E. A. and Chen, L. 1982 Phys. Fluids 25502

[3] Hahm T. S. 1988 Phys. Fluids 312673

[4] Brizard, A. and Hahm, T. S. 2007 Rev. Modern Physics 2 421-468

[5] Parra, F. I. and Calvo, I. 2011 Plasma Phys. Control. Fusion 53045001

[6] Faber, B. et al. 2015 Phys. Plasmas 22, 072305

[7] Nadeem, M., Rafiq, T., \& Persson, M. 2001 Phys. Plasmas 8(10), 4375

[8] Kornilov, V. et al. 2004 Phys. Plasmas 11 3196-3202

[9] Sánchez E. et al. Spatial localization of electrostatic microinstabilities in the TJ-II stellarator. 43rd European Physical Society Conference on Plasma Physics. Leuven, Belgium (2016) (to be published)

[10] Xanthopoulos, P. Mynick, H. E., Helander, P. et al. 2014 Physical Review Letters 113155001

[11] Helander, P et al. 2012 Plasma Physics and Controlled Fusion 12, 124009

[12] Helander, P. et al. 2015 Nuclear Fusion 5 (55), 053030

[13] Villard, L.; Bottino, A.; Sauter, O.; Vaclavik, J.2002 Physics of Plasmas 6 (9), 2684

[14] Riemann, J., Kleiber, R. \& Borchardt, M. 2016 Plasma Physics and Controlled Fusion 58(7), 74001

[15] Mischenko, A., et al. 2008 Physics of Plasmas 15(7), 72309

[16] Plunk, G. Xanthopoulos, P. \& Helander, P. 2017 Physical Review Letters 118(10)

[17] Jost, G. et al. 2001 Physics of Plasmas 8(7), 3321

[18] Faber B. et al 2018 J. of Plasma Phys. 84, 905840503.

[19] Sánchez, E; Kleiber, R; Hatzky, R; Borchardt, M; Monreal, et al. 2013 Plasma Physics and Controlled Fusion 55, 014015

[20] Sánchez E., Monreal P., Calvo I., Kleiber R. Relaxation of zonal flows in stellarators: influence of the 
magnetic configuration. 42th European Physical Society Conference on Plasma Physics. Lisbon, Portugal. 2015.

[21] Monreal, P. et al. 2016 Plasma Phys. Control. Fusion 58

[22] Monreal, P. et al. 2017 Plasma Phys. Control. Fusion 5 9, 065005

[23] Alonso, J. A. et al. 2017 Phys. Rev. Lett. 118.18, p. 185002

[24] Sánchez E., Calvo I., Velasco J. L. R Kleiber et al. 2018. Plasma Phys. Control. Fusion 60, 094003

[25] Kornilov, V. Kleiber, R. and Hatzky, R. 2004 Nuclear Fusion 45238

[26] Kauffmann, K. et al. 2010 Journal of Physics: Conference Series 260012014

[27] Helander, P. and Simakov A. N. 2008 Phys. Rev. Lett. 101 145003

[28] Lee. W. W. 1987 Journal of Computational Physics 72243.

[29] Lin, Z. and Lee, W. W. 1995 Physical Review E 52(5), 5646.

[30] Grimm R C, Greene J M, Johnson J L. 1976 Methods in Comp. Phys. 16253

[31] Hirshman S. P. et al. Phys. 1983 Fluids 263553.

[32] Kleiber, R. et al. 2006 Theory of Fusion Plasmas. 871 AIP Conference proceedings 1. AIP, 2006, pp. 136-146.

[33] Kleiber, R. and Hatzky, R. 2012 Comput. Phys. Commun. 183 305-308

[34] Borchardt M, Kleiber R , and Hackbusch W. 2012 Journal of Computational Physics 231 6207-6212

[35] Fernandez, A., Kasparev, W. and Likin, K. 2001 International Journal of Infrared and Millimeter Waves 22,649

[36] Liniers, M. and Alonso, J. 1998 Fusion Technology 1, 307

[37] Happel, T. et al. 2009 Rev. Sci. Instrum. 80, 073502

[38] Castejon F., Cappa A., Tereshchenko M. and Fernandez A. 2008 Nuclear Fusion 48, 075011

[39] Estrada, T. et al. Turbulence and radial electric field asymmetries measured at TJ-II plasmas. 27th IAEA Fusion Energy Conference, EX/P1-9, Ahmedabad, India, (2018) (to be submitted to Nuclear Fusion)

[40] Bañon Navarro A. et al. 2016 Phys. Plasmas 23, 042312.

[41] Velasco J L et al. 2012 Phys. Rev. Lett. 109135003

[42] van Milligen B. Ph., et al. 2011 Rev. Sci. Instr. 82073503

[43] Estrada T. et al. 2001 Plasma Phys. Control. Fusion 43, $1535-1545$

[44] Herranz J., Castejón F., Pastor I., McCarthy K. 2003 Fusion Engineering and Design 65: 525 - 536.

[45] Fontdecaba J.M. et al. 2004 Fusion Sci. Tech. 46.

[46] Branas B., Tafalla D., Tabares F. L., Ortiz P 2001 Review of Sci. Instr. 72: 602-606.

[47] Guzmán F. Taberés F., Tafalla D., Cortés I.G.Balbín R. 2009 Journal of Nuclear Materials 390.

[48] Tabares F. L., Tafalla D., Ferreira J. A., Guzman F. 2010 Review of Sci. Instr. 81: 10D708.

[49] de la Luna E. et al. 2001 Review of Sci. Instr. 72, 379

[50] Fontdecaba J. M. et al. 2010 Plasma and Fusion Research $5, \mathrm{~S} 2085$

[51] Sánchez E. at al. Characterization of instabilities in TJ-II: experiment vs numerical simulations. 21st International Stellarator/Heliotron Workshop, Kyoto, Japan (2017).

[52] Hirshmann S. P. et al. 1986 Phys. Fluids 29, 2951

[53] M. G. Shats, H. Xia, and H. Punzmann, 2005 Phys. Rev. E 71, 046409

[54] J L Velasco et al. 2011 Plasma Phys. Control. Fusion 53 115014

[55] Sánchez E. et al. Simulation of electrostatic instabilities in a heliac configuration, 41th European Physical Society Conference on Plasma Physics. Berlin, Germany. 2014.

[56] Fivaz M. et al. 1998 Computer Phys. Comm. 111 1-3, $27-47$.

[57] Hatzky R. et al. 2002 Phys. Plasmas 9 1-3, 898

[58] Ryter, F et al. 2005 Physical Review Letters 8 (95), 85001

[59] Estrada T. et al., 2009 Plasma Phys. Control. Fusion 51,
124015

[60] Happel T. et al., 2015 Phys. Plasmas 22, 032503

[61] Plunk G. et al. 2017 J. Plasma Phys. 83, 715830404

[62] Dickinson D. et al 2014 Physics of Plasmas 21, 010702

[63] García-Regaña J.M. et al., 2018 Plasma Phys. Control. Fusion 60, 104002

[64] Vermare L. et al., 2011 Phys. Plasmas 18, 012306

65] Losada U. et al. 2018. Plasma Phys. Control. Fusion 60, 7

[66] Nakata M. et al 2017 Phys. Rev. Lett.118, 16, 165002. 